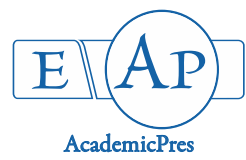

\title{
Effects of Weed Density and Distance on the Growth and Yield of Two Okra (Abelmoscus esculentus) Varieties
}

\section{Wasiu Olanrewaju OPADOKUN*, Kehinde Stephen OLORUNMAIYE}

\author{
University of Ilorin, Faculty of Life Sciences, Department of Plant Biology, Nigeria; Waseopas@gmail.com (*correspondingauthor)
}

\begin{abstract}
A field experiment was conducted at the botanical garden of the University of Ilorin to determine the effects of weed density and distance on the growth and yield of two varieties of okra (NHAe-40 days and Kirikoi). The experiment was a complete randomized block design with eleven treatments and three replicates. The treatment details include $\mathrm{T}_{0}=\mathrm{No}_{\mathrm{s}}$ weed, $\mathrm{T}_{1}=$ One weed per plant $\mathrm{T}_{2}=$ Two weeds per plant, $\mathrm{T}_{3}=$ Three weeds per plant, $\mathrm{T}_{4}=$ Four weeds per plant, $\mathrm{T}_{5}=$ Five weeds per plant, $\mathrm{T}_{6}=$ Six weeds per plant, $\mathrm{T}_{7}=$ Seven weeds per plant, $\mathrm{T}_{8}=$ Eight weeds per plant, $\mathrm{T}_{9}=$ Nine weeds per plant and $\mathrm{T}_{10}$ $=$ Ten weeds per plant. Data were collected on plant height, number of leaves, stem girth, shoot Fresh and dry weight as well as yield parameters was carried out at the end of the experiment. The result depicts a significant decrease in the vegetative growth and yield parameters with increasing weed density in both varieties. Irrespective of the weed distance, all yield parameters decreased significantly with increasing number of co-habiting weeds except for plant co-habited with one weed at $20 \mathrm{~cm}$ and $30 \mathrm{~cm}$ weed distance which was significantly the same with the control in both varieties. The result is an indication that absence of weed or early control of weed should be done at the early weeks of growth before the onset of flowering if maximum production is expected.
\end{abstract}

Keywords: competition; density; distance; okra; weed

\section{Introduction}

Okra (Abelmoscus esculentus (L). Moench) is an economically important vegetable crop grown in tropical and subtropical part of the world including India, Sudan, Parkistan, Ghana, Egypt, Benin Saudi Arabia, Mexico and Cameroon with the largest area in India followed by Nigeria (Saifullah and Rabbani, 2009). It belongs to the Malvaceae family (Kashif et al., 2008). Globally, it is grown for its immature pods consumed as fried or boiled vegetable or may be added to salad, soups and stew (Kashif et al., 2008). In Nigeria, the fruits are used in making soup, salad and for flavoring when dried and powdered. The tender fruits contain minerals especially calcium, magnesium, Iron and phosphorus, protein, vitamin $\mathrm{A}$ and $\mathrm{C}$ including riboflavin as well as high mucilage (Poggio, 2005). Mature okra seed are good sources of protein and oil and it has been known for its high nutritional quality. Okra cultivation is often limited by several factors including inadequate soil fertility, pest and diseases infestation, weed competition and poor agronomic practices such as sub-optimal plant density, inappropriate planting date to mention but a few (Chadha, 2002) however, of all the constraint to its production, weed competition constitute a major case of yield loss in okra. Euphorbia heterophylla is a perennial herb which has been identified as an important weed for most waste and farmlands, railway banks throughout the tropical and subtropical regions of the world (Falodun et al., 2006). In Nigeria the weed have been reported in association with cowpea (Olorunmaiye and Ogunfolaji, 2002), popcorn maize (Saka et al., 2013) and several vegetable crops including okra (Panthinige et al., 2008). It has been reported that optimum weed control is the key element for higher yield in okra plant growth and yield are affected by weed competition (Amjad et al., 2002). The present study therefore seeks to examine the effects of $E$. heterophylla weed distance from the crop of interest as well as the weed density on the growth and yield of two okra varieties (Abelmoschus esculentus).

\section{Materials and Methods}

The experiment was carried out at University of Ilorin Botanical garden which lies in the southern guinea savanna belt of Nigeria with an annual rainfall of $1200 \mathrm{~mm}$ and temperature range of $33-34^{\circ} \mathrm{C}$ and a distinct dry season. The experimental soil is sandy loam with a $\mathrm{pH}$ of 7.8 The experimental design is a split plot completely randomized block design with two main plots and three sub-plots, each sub-plot comprises of eleven treatments and three 
430

replicates including $\mathrm{T}_{0}=$ No weed, $\mathrm{T}_{1}=1$ weed per plant, $\mathrm{T}_{2}=2$ weeds per plant, $\mathrm{T}_{3}=3$ weeds per plant, $\mathrm{T}_{4}=4$ weeds per plant, $T_{5}=5$ weeds per plant, $T_{6}=6$ weeds per plant, $T_{7}$ $=7$ weeds per plant, $T_{8}=8$ weeds per plant, $T_{9}=9$ weeds per plant, $T_{10}=10$ weeds per plant. Five seeds each of the two varieties of Okra ('NHAe-40 days' and 'Kirikoi') procured from an Agricultural store in Ilorin, were sown per hole with 1 to 10 E. heterophylla seeds planted at $20 \mathrm{~cm}$ away from the okra. The same procedure as above were used for the $30 \mathrm{~cm} \mathrm{E}$. heterophylla distance while the same were also done for the weed density however, for this group, the weeds were planted in a concentric manner around the crop irrespective of their distance from the crop. All weeds found on the experimental field were removed on site except for $E$. heterophylla.

Morphological growth attributes such as plant height, number of leaves, stem girth and leaves area were estimated at an interval of two weeks throughout the period of cultivation. Reproductive parameters including average number of fruit, average groove number, average pod length, pod girth and fresh and dry pod weight were determined at harvest. Data collected were subjected to analysis of variance (ANOVA) using Statistical Package of Social Science (SPSS) and means were separated using Duncan's Multiple Range Test (DMRT) at 5\% level of probability.

\section{Results and Discussion}

Euphorbia heterophylla weed density and distance significantly $(\mathrm{p}<0.05)$ affect the plant height of the two varieties of okra considered in this study (Table 1). Significant highest plant height was observed in weed free check (control) and those cohabited with one weed. There was a progressive reduction in plant height of okra as the density of weeds increased in the two varieties when compared to the control treatment with the shortest plants obtained in those co-habited with ten weeds irrespective of the distance (Table 1). The tallest plants obtained in the control and those co-habited with one weed could be attributed to less weed competition with the okra plant for nutrients, light, water and space (Reddy and Whiting, 2000; Odelaye et al., 2007). The general reduction in plant height with increasing weed density could be attributed to an intense weed competition with increasing number of weed per plant stand (Barrentine and Oliver, 1977; Rao, 2000). This result also agrees with findings of Ayeni and Oyenka (1992) that attributed the stronger depressive effects of weed on soybean plant to a longer duration of weed interference. Number of leaves increased steadily with the age of okra plants from 4-6WAP beyond which a decrease in leaf number was observed till harvest. The reduction in the number of leaf of both varieties could be attributed to an increase in the number and density of weed (E. heterophylla) which ultimately result in a reduction in the photosynthetic activities of the crop due to competition. This is aligns with the work of (RIzzardi, 2004; Carvalho et al., 2010) who reported a significant decrease in growth parameters such as plant height, number of leaves, dry matter accumulation and macronutrient accumulation in soybean co-habited with $E$. .heterophylla were reduced with increasing weed density. Significant highest stem girth in both okra varieties were observed in the control treatment followed by those cohabited with one weeds with the lowest stem diameter was recorded in those cohabited with ten weeds. The present findings was in consonance with (Smith and Ojo, 2007) they observed the maximum stem diameter of okra where weeding was done at early week of planting. In both varieties weed free check (control) recorded a significant highest $(p<0.05)$ leaf area when compared to all other plants co-habited with $E$. heterophylla weed. The highest leaf area of the two varieties in the control treatments in both varieties could be adduced to increased cell division and elongation on an account of efficient utilization of available resources since there was no weed competition at all. Ibe $e t$ al. (2005) reported efficient resources utilization in weed free plants which resulted in higher yield. Askew et al. (2002) conducted field trial and reported that controlling weeds and leaser competition within the plant community could result in utilization of the available resources efficiently. Similarly, significantly higher dry biomass accumulation was observed in the Weed free check (control) This is in tandem with the work of (Rizzardi 2004; Carvalho et al., 2010) in a soybean study in coexistence with $E$. heterophylla, they reported a reduced growth (plant height, number of leaves, dry matter accumulation) and macronutrient accumulation of in soybean plant with increasing $E$. heterophylla weed density.

In both varieties of okra, reproductive performance of the various weed treatments of 20 and $30 \mathrm{~cm}$ weed distance and weed density was considered and the control treatment recorded the highest yield attributes ( Tables 6 and 7) such as in number of fruits, groove number, pod length, pod girth and the pod fresh and dry weight. The densities of $E$. heterophylla reduced the yield of okra in both varieties compared with the control in terms of number of fruits, number of groove, pod length, pod girth, fresh and dry pod weight. This observed reduction in yield agreed with the finding of Olorunmaiye and Ogunfolaji (2002) who reported reduction in cowpea yield with increase in $E$. heterophylla population. Askew et al. (2002) conducted field trial and reported that controlling weeds and leaser competition within the plant community could result in utilization of the available resources efficiently, which is in turn reflected in higher yield. Moreover, the availability of adequate soil moisture, nutrients and other growth factors due to less weed competition also contributed to optimum okra yield, which is similar to the study conducted by Reddy and Whiting (2002).

\section{Conclusions}

The decrease in growth and yield characteristics of okra is a function of increasing number of Euphorbia heterophylla stands co-habited with the okra plant. It should be noted that plant co-habited with one stand of $E$. heterophylla weed irrespective of the distance from the plant had significantly the same effect on the growth and yield performance of okra when compared to the weed free check. Therefore, $E$. heterophylla weed co-existence should be completely avoided where possible for optimum growth and yield of okra plant, where a complete removal of the weed is economically not feasible, the weed density should not exceed one weed per plant stand and the weed distance from the plant should not be less than $30 \mathrm{~cm}$ away from the plant

\section{Conflict of Interest}

The authors declare that there are no conflicts of interest related to this article. 
Table 1. Effects of E. heterophylla weeds density and distance on the plant height of A. esculentus (Var. 'Kirikoi' and 'NHAe-40days')

\begin{tabular}{|c|c|c|c|c|c|c|c|c|c|}
\hline & \multirow{2}{*}{ Treatments } & \multicolumn{4}{|c|}{$\begin{array}{c}\text { Var. 'Kirikoi' } \\
\end{array}$} & \multicolumn{4}{|c|}{ Var. 'NHAe-40 days' } \\
\hline & & 2 WAP & $4 \mathrm{WAP}$ & $6 \mathrm{WAP}$ & $8 \mathrm{WAP}$ & 2 WAP & $4 \mathrm{WAP}$ & $6 \mathrm{WAP}$ & $8 \mathrm{WAP}$ \\
\hline \multirow{11}{*}{$\begin{array}{c}20 \mathrm{~cm} \\
\text { weed } \\
\text { distance }\end{array}$} & $\mathrm{T}_{0}$ & $7.83 \pm 0.17^{\mathrm{a}}$ & $15.53 \pm 0.91^{2}$ & $32.33 \pm 1.67^{\mathrm{a}}$ & $37.67 \pm 1.09^{2}$ & $8.07 \pm 0.46^{2}$ & $15.83 \pm 0.44^{2}$ & $25.13 \pm 0.55^{a}$ & $32.57 \pm 0.83^{\mathrm{a}}$ \\
\hline & $T_{1}$ & $6.13 \pm 0.59^{\mathrm{b}}$ & $15.67 \pm 0.73^{2}$ & $31.20 \pm 1.85^{a}$ & $33.13 \pm 1.54^{\mathrm{b}}$ & $7.70 \pm 0.21^{\mathrm{ab}}$ & $15.33 \pm 0.12^{\mathrm{ab}}$ & $24.63 \pm 0.27^{\mathrm{ab}}$ & $31.87 \pm 0.28^{\mathrm{ab}}$ \\
\hline & $\mathrm{T}_{2}$ & $7.00 \pm 0.29^{\mathrm{ab}}$ & $15.67 \pm 1.01^{2}$ & $29.67 \pm 1.20^{4}$ & $31.93 \pm 2.08^{\mathrm{bc}}$ & $3.70 \pm 0.38^{f}$ & $10.90 \pm 0.42^{\mathrm{dc}}$ & $19.70 \pm 0.72^{f}$ & $27.07 \pm 0.75^{\text {cf }}$ \\
\hline & $\mathrm{T}_{3}$ & $6.53 \pm 0.26^{\mathrm{b}}$ & $15.23 \pm 0.67^{2}$ & $28.93 \pm 1.19^{a}$ & $32.37 \pm 1.50^{\mathrm{bc}}$ & $7.07 \pm 0.09 b^{c}$ & $14.67 \pm 0.17^{c}$ & $23.30 \pm 0.44^{\mathrm{bc}}$ & $30.13 \pm 0.58^{\mathrm{bc}}$ \\
\hline & $\mathrm{T}_{4}$ & $6.70 \pm 0.21^{\mathrm{ab}}$ & $13.53 \pm 1.22^{\mathrm{ab}}$ & $21.83 \pm 1.09^{\mathrm{bc}}$ & $31.10 \pm 1.47^{\mathrm{bc}}$ & $7.07 \pm 0.24 b^{\mathrm{c}}$ & $14.37 \pm 0.13^{\mathrm{bc}}$ & $22.83 \pm 0.17^{\mathrm{cd}}$ & $29.70 \pm 0.36^{\mathrm{cd}}$ \\
\hline & $\mathrm{T}_{5}$ & $6.73 \pm 0.64^{\mathrm{ab}}$ & $12.60 \pm 1.07 \mathrm{~b}^{c}$ & $21.03 \pm 2.04^{\mathrm{bc}}$ & $30.13 \pm 1.70^{\text {bed }}$ & $6.63 \pm 0.24^{c}$ & $13.40 \pm 0.59^{c}$ & $21.50 \pm 0.87^{\mathrm{dc}}$ & $28.17 \pm 1.10^{\text {cdc }}$ \\
\hline & $\mathrm{T}_{6}$ & $4.33 \pm 0.33^{\mathrm{bc}}$ & $12.53 \pm 0.32 b^{c}$ & $19.53 \pm 1.35^{c}$ & $28.07 \pm 1.01^{\mathrm{cde}}$ & $6.73 \pm 0.27^{\circ}$ & $13.47 \pm 0.32^{c}$ & $21.27 \pm 0.15^{c}$ & $27.80 \pm 0.17^{\mathrm{de}}$ \\
\hline & $\mathrm{T}_{7}$ & $4.93 \pm 0.47^{c}$ & $10.63 \pm 0.95^{\mathrm{cd}}$ & $24.83 \pm 0.73^{\mathrm{b}}$ & $26.30 \pm 1.20^{\text {cf }}$ & $5.37 \pm 0.52^{d}$ & $11.67 \pm 0.33^{d}$ & $19.27 \pm 0.12^{f}$ & $25.73 \pm 0.13^{\text {fy }}$ \\
\hline & $\mathrm{T}_{8}$ & $4.33 \pm 0.44^{\mathrm{cd}}$ & $10.73 \pm 0.27^{\mathrm{cd}}$ & $19.90 \pm 0.86^{c}$ & $26.47 \pm 0.84^{\text {def }}$ & $5.17 \pm 0.32^{\mathrm{d}}$ & $10.77 \pm 0.39^{\text {de }}$ & $18.30 \pm 0.20^{f_{g}}$ & $24.57 \pm 0.38^{8}$ \\
\hline & $\mathrm{T}_{9}$ & $4.37 \pm 0.30^{\text {cd }}$ & $9.57 \pm 0.78^{c}$ & $19.50 \pm 0.76^{c}$ & $24.47 \pm 0.69^{c f}$ & $4.73 \pm 0.07^{\mathrm{de}}$ & $10.50 \pm 0.29^{c}$ & $17.63 \pm 0.63^{\mathrm{gh}}$ & $23.77 \pm 0.86^{\mathrm{gh}^{h}}$ \\
\hline & $T_{10}$ & $3.37 \pm 0.19^{\mathrm{d}}$ & $7.20 \pm 0.23^{\mathrm{c}}$ & $18.63 \pm 0.73^{c}$ & $23.23 \pm 1.65^{f}$ & $4.10 \pm 0.12^{f}$ & $9.33 \pm 0.34^{f}$ & $16.27 \pm 0.62^{\mathrm{h}}$ & $22.30 \pm 0.83^{h}$ \\
\hline \multirow{11}{*}{$\begin{array}{c}30 \mathrm{~cm} \\
\text { weed } \\
\text { distance }\end{array}$} & $\mathrm{T}_{0}$ & $6.57 \pm 0.15^{a}$ & $15.33 \pm 0.33^{\mathrm{ab}}$ & $26.00 \pm 1.50^{\mathrm{a}}$ & $35.93 \pm 0.92^{a}$ & $8.57 \pm 0.34^{a}$ & $16.33 \pm 0.42^{a}$ & $25.63 \pm 0.73^{a}$ & $33.07 \pm 0.80^{a}$ \\
\hline & $\mathrm{T}_{1}$ & $5.90 \pm 0.35^{\mathrm{a}}$ & $12.67 \pm 0.60^{\mathrm{bc}}$ & $25.83 \pm 1.74^{a}$ & $31.73 \pm 0.73^{\mathrm{ab}}$ & $7.80 \pm 0.42^{\mathrm{ab}}$ & $15.43 \pm 0.66^{\mathrm{ab}}$ & $24.73 \pm 0.74^{\mathrm{ab}}$ & $31.97 \pm 0.72^{\mathrm{ab}}$ \\
\hline & $\mathrm{T}_{2}$ & $5.97 \pm 0.23^{\mathrm{a}}$ & $15.50 \pm 0.76^{a}$ & $21.53 \pm 2.11^{\mathrm{b}}$ & $31.20 \pm 2.84^{\mathrm{ab}}$ & $7.17 \pm 0.09^{\mathrm{bc}}$ & $14.37 \pm 0.23^{\text {bcd }}$ & $23.17 \pm 0.41^{\mathrm{bc}}$ & $30.53 \pm 0.47^{\mathrm{bc}}$ \\
\hline & $\mathrm{T}_{3}$ & $6.13 \pm 0.12^{\mathrm{a}}$ & $12.67 \pm 0.93^{\mathrm{bc}}$ & $21.03 \pm 0.53^{\mathrm{b}}$ & $31.10 \pm 1.22^{\mathrm{ab}}$ & $6.90 \pm 0.26^{\text {bcd }}$ & $14.50 \pm 0.40^{\mathrm{bc}}$ & $23.13 \pm 0.52^{\mathrm{bc}}$ & $29.97 \pm 0.69^{b x}$ \\
\hline & $\mathrm{T}_{4}$ & $6.20 \pm 0.50^{2}$ & $11.83 \pm 0.44^{c^{d d}}$ & $19.23 \pm 0.50^{b c}$ & $28.97 \pm 1.18^{\text {abc }}$ & $6.30 \pm 0.06^{\mathrm{dec}}$ & $13.60 \pm 0.15^{\mathrm{cdc}}$ & $22.07 \pm 0.12^{\mathrm{cd}}$ & $28.93 \pm 0.54^{\mathrm{cd}}$ \\
\hline & $\mathrm{T}_{5}$ & $6.00 \pm 0.15^{\mathrm{a}}$ & $13.23 \pm 0.90^{\mathrm{abc}}$ & $20.67 \pm 0.93^{b}$ & $27.63 \pm 1.41^{\mathrm{abc}}$ & $6.17 \pm 0.59^{\mathrm{dc}}$ & $12.93 \pm 0.87^{\mathrm{dc}}$ & $21.03 \pm 1.18^{\mathrm{de}}$ & $27.70 \pm 1.39^{\mathrm{de}}$ \\
\hline & $\mathrm{T}_{6}$ & $5.77 \pm 0.35^{a}$ & $13.00 \pm 1.32^{\mathrm{abc}}$ & $18.73 \pm 1.77^{\mathrm{bc}}$ & $25.77 \pm 1.39^{\mathrm{bc}}$ & $5.97 \pm 0.34^{\mathrm{dc}}$ & $12.70 \pm 0.70^{\mathrm{c}}$ & $20.50 \pm 0.57^{\mathrm{dc}}$ & $27.03 \pm 0.39^{\mathrm{de}}$ \\
\hline & $\mathrm{T}_{7}$ & $6.10 \pm 0.40^{\mathrm{a}}$ & $12.10 \pm 1.16^{\mathrm{c}}$ & $16.17 \pm 1.20^{\circ}$ & $26.20 \pm 0.92^{\mathrm{bc}}$ & $5.77 \pm 0.32^{c}$ & $12.07 \pm 0.62^{\mathrm{c}}$ & $19.67 \pm 0.64^{\mathrm{ct}^{f}}$ & $26.13 \pm 0.82^{c f}$ \\
\hline & $\mathrm{T}_{8}$ & $4.13 \pm 0.12^{\mathrm{b}}$ & $10.83 \pm 1.17^{\mathrm{cdc}}$ & $15.20 \pm 0.85^{d}$ & $27.30 \pm 1.63^{\text {alc }}$ & $4.70 \pm 0.32^{\mathrm{f}}$ & $10.30 \pm 0.23^{f}$ & $17.83 \pm 0.38^{\mathrm{fg}}$ & $24.10 \pm 0.20^{\text {f8 }}$ \\
\hline & $\mathrm{T}_{9}$ & $4.00 \pm 0.20^{\mathrm{b}}$ & $9.40 \pm 0.42^{\mathrm{de}}$ & $16.33 \pm 0.33^{c}$ & $22.93 \pm 1.56^{\mathrm{cd}}$ & $4.63 \pm 0.20^{f}$ & $10.40 \pm 0.21^{\mathrm{f}}$ & $17.53 \pm 0.57 \mathrm{~g}$ & $23.67 \pm 0.78^{8}$ \\
\hline & $\mathrm{T}_{10}$ & $4.20 \pm 0.56^{b}$ & $9.17 \pm 0.44^{c}$ & $11.70 \pm 1.40^{\mathrm{d}}$ & $19.30 \pm 0.93^{\mathrm{d}}$ & $4.57 \pm 0.17^{f}$ & $9.80 \pm 0.30^{f}$ & $16.73 \pm 0.47 \mathrm{~g}$ & $22.77 \pm 0.62^{\mathrm{g}}$ \\
\hline \multirow{11}{*}{$\begin{array}{l}\text { Weed } \\
\text { density }\end{array}$} & $\mathrm{T}_{0}$ & $5.77 \pm 0.33^{a}$ & $11.00 \pm 0.92^{2}$ & $18.17 \pm 0.60^{2}$ & $19.80 \pm 0.17^{\mathrm{a}}$ & $7.87 \pm 0.12^{\mathrm{a}}$ & $15.63 \pm 0.12^{\mathrm{a}}$ & $24.93 \pm 0.43^{a}$ & $32.37 \pm 0.57^{\mathrm{a}}$ \\
\hline & $\mathrm{T}_{1}$ & $5.13 \pm 0.22^{b}$ & $10.83 \pm 0.60 \mathrm{a}$ & $17.40 \pm 0.70^{a}$ & $18.77 \pm 0.89^{\mathrm{ab}}$ & $5.90 \pm 0.21^{b}$ & $13.53 \pm 0.39^{b}$ & $22.83 \pm 0.52^{b}$ & $30.07 \pm 0.52^{b}$ \\
\hline & $\mathrm{T}_{2}$ & $4.83 \pm 0.23^{\mathrm{bc}}$ & $11.00 \pm 1.00^{2}$ & $16.00 \pm 0.58 \mathrm{~b}^{\mathrm{c}}$ & $18.50 \pm 0.83^{\mathrm{ab}}$ & $5.47 \pm 0.27^{\mathrm{bc}}$ & $12.67 \pm 0.42^{\mathrm{bc}}$ & $21.47 \pm 0.39^{\mathrm{bc}}$ & $28.83 \pm 0.50^{\mathrm{bcc}}$ \\
\hline & $\mathrm{T}_{3}$ & $4.90 \pm 0.06^{\mathrm{bc}}$ & $9.83 \pm 0.33^{\text {ab }}$ & $15.97 \pm 0.03 b^{c}$ & $17.60 \pm 0.32^{\mathrm{ab}}$ & $5.23 \pm 0.26^{\mathrm{c}}$ & $12.83 \pm 0.24^{\mathrm{bc}}$ & $21.47 \pm 0.69^{\mathrm{bc}}$ & $28.30 \pm 0.78^{\text {bec }}$ \\
\hline & $\mathrm{T}_{4}$ & $4.73 \pm 0.12^{\text {bcd }}$ & $9.80 \pm 0.61^{\mathrm{ab}}$ & $15.33 \pm 0.44^{c}$ & $16.93 \pm 1.62^{\mathrm{bcd}}$ & $5.10 \pm 0.12^{\mathrm{cd}}$ & $12.40 \pm 0.00^{\mathrm{bc}}$ & $20.87 \pm 0.03^{\text {cd }}$ & $27.73 \pm 0.42^{\text {de }}$ \\
\hline & $\mathrm{T}_{5}$ & $4.53 \pm 0.15^{\text {bcd }}$ & $9.83 \pm 0.44^{\mathrm{bb}}$ & $14.33 \pm 0.44^{c}$ & $15.90 \pm 0.95^{\mathrm{cdc}}$ & $5.07 \pm 0.09^{\mathrm{de}}$ & $11.83 \pm 0.39^{\mathrm{cd}}$ & $19.93 \pm 0.66^{\mathrm{de}}$ & $26.60 \pm 0.90^{\text {det }}$ \\
\hline & $\mathrm{T}_{6}$ & $4.80 \pm 0.26^{6 \mathrm{bcd}}$ & $10.00 \pm 0.29^{\mathrm{ab}}$ & $13.50 \pm 0.29^{\mathrm{dc}}$ & $4.90 \pm 0.90^{\mathrm{dc}}$ & $5.00 \pm 0.29^{\mathrm{de}}$ & $11.73 \pm 0.55^{\mathrm{cd}}$ & $19.53 \pm 0.54^{\mathrm{dc}}$ & $26.07 \pm 0.64^{\mathrm{ff}}$ \\
\hline & $\mathrm{T}_{7}$ & $4.57 \pm 0.09^{\mathrm{bcd}}$ & $9.67 \pm 0.44^{\mathrm{ab}}$ & $12.73 \pm 0.62^{d k}$ & $14.33 \pm 0.35^{\text {def }}$ & $4.50 \pm 0.17^{\mathrm{def}}$ & $10.80 \pm 0.38^{\mathrm{dc}}$ & $18.40 \pm 0.35^{\mathrm{cf}}$ & $24.87 \pm 0.54^{6 \mathrm{~g}}$ \\
\hline & $\mathrm{T}_{8}$ & $4.43 \pm 0.07^{\mathrm{cd}}$ & $8.57 \pm 0.55^{c}$ & $13.07 \pm 0.57^{\mathrm{dc}}$ & $14.30 \pm 0.40^{\text {def }}$ & $4.43 \pm 0.27^{\mathrm{cf}}$ & $10.03 \pm 0.49 \mathrm{ef}$ & $17.57 \pm 0.44^{\mathrm{f}}$ & $23.83 \pm 0.38^{g^{h}}$ \\
\hline & $\mathrm{T}_{9}$ & $4.40 \pm 0.12^{\mathrm{bc}}$ & $8.20 \pm 0.15^{c}$ & $12.67 \pm 0.67^{\mathrm{dc}}$ & $13.37 \pm 0.33^{\text {cf }}$ & $4.03 \pm 0.13^{8}$ & $9.80 \pm 0.25^{\mathrm{cf}}$ & $16.93 \pm 0.62^{\mathrm{fg}}$ & $23.07 \pm 0.84^{\mathrm{gh}^{\mathrm{h}}}$ \\
\hline & $\mathrm{T}_{10}$ & $4.20 \pm 0.15^{\mathrm{d}}$ & $8.27 \pm 0.39^{c}$ & $11.83 \pm 0.67^{\mathrm{c}}$ & $12.13 \pm 0.97^{f}$ & $3.70 \pm 0.15^{g}$ & $8.93 \pm 0.39^{f}$ & $15.87 \pm 0.67^{8}$ & $21.90 \pm 0.87^{\mathrm{h}}$ \\
\hline
\end{tabular}

Values represent Means \pm SEM, $\mathrm{n}=3$, values with same superscript along the column are significantly the same at $\mathrm{p}<0.05 ; \mathrm{T}_{0}=\mathrm{control}, \mathrm{T}_{1}=1$ weed, $\mathrm{T}_{1}=1$ weed plant ${ }^{-1}$ 10 weed plant ${ }^{-1}$.

Table 2. Effects of E. heterophylla weeds density and distance on the number of leaves of $A$. esculentus (Var. 'Kirikoi' and 'NHAe- 40 days')

\begin{tabular}{|c|c|c|c|c|c|c|c|c|c|}
\hline & \multirow{2}{*}{ Treatments } & \multicolumn{4}{|c|}{ Var. 'Kirikoi' } & \multicolumn{4}{|c|}{ Var. 'NHAe-40days' } \\
\hline & & $2 \mathrm{WAP}$ & $4 \mathrm{WAP}$ & $6 \mathrm{WAP}$ & $8 \mathrm{WAP}$ & $2 \mathrm{WAP}$ & $4 \mathrm{WAP}$ & $6 \mathrm{WAP}$ & $8 \mathrm{WAP}$ \\
\hline \multirow{11}{*}{$\begin{array}{c}20 \mathrm{~cm} \\
\text { weed } \\
\text { distance }\end{array}$} & $\mathrm{T}_{0}$ & $4.00 \pm 0.00^{2}$ & $6.00 \pm 0.00^{2}$ & $7.33 \pm 0.33^{a}$ & $6.33 \pm 0.33^{a}$ & $3.33 \pm 0.33^{a}$ & $4.67 \pm 0.67^{2}$ & $7.33 \pm 0.33^{a}$ & $5.67 \pm 0.33^{a}$ \\
\hline & $T_{1}$ & $3.67 \pm 0.33^{\text {b }}$ & $5.67 \pm 0.33^{\text {b }}$ & $7.00 \pm 0.58^{\mathrm{b}}$ & $5.33 \pm 0.33^{\mathrm{b}}$ & $3.33 \pm 0.33^{2}$ & $4.67 \pm 0.33^{2}$ & $7.33 \pm 0.33^{a}$ & $5.33 \pm 0.33^{\text {b }}$ \\
\hline & $\mathrm{T}_{2}$ & $3.33 \pm 0.33^{\mathrm{abc}}$ & $5.33 \pm 0.67^{\mathrm{ab}}$ & $6.33 \pm 0.67^{\mathrm{at} x}$ & $5.33 \pm 0.33 b$ & $3.33 \pm 0.33^{a}$ & $4.67 \pm 0.33^{\mathrm{a}}$ & $7.00 \pm 0.00^{\mathrm{ab}}$ & $5.00 \pm 0.00^{\mathrm{abc}}$ \\
\hline & $\mathrm{T}_{3}$ & $3.67 \pm 0.33^{\text {ab }}$ & $5.33 \pm 0.6^{\mathrm{zb}}$ & $6.33 \pm 0.88^{\mathrm{alc}}$ & $5.33 \pm 0.33^{\mathrm{b}}$ & $3.00 \pm 0.00^{2}$ & $4.33 \pm 0.33^{\mathrm{b}}$ & $7.00 \pm 0.00^{-\mathrm{b}}$ & $5.00 \pm 0.00^{\mathrm{bcc}}$ \\
\hline & $\mathrm{T}_{4}$ & $3.33 \pm 0.67^{\mathrm{abc}}$ & $5.33 \pm 0.33^{\mathrm{ab}}$ & $5.67 \pm 0.33^{\text {alkd }}$ & $4.67 \pm 0.33^{\mathrm{bc}}$ & $3.33 \pm 0.33^{a}$ & $5.00 \pm 0.00^{2}$ & $6.33 \pm 0.33^{\mathrm{bc}}$ & $4.67 \pm 0.33^{\text {bcd }}$ \\
\hline & $T_{5}$ & $3.33 \pm 0.33^{\text {abc }}$ & $4.67 \pm 0.33^{\text {abc }}$ & $6.00 \pm 0.00^{\text {alkad }}$ & $4.67 \pm 0.33^{\mathrm{bc}}$ & $2.67 \pm 0.33^{a}$ & $4.33 \pm 0.33^{\mathrm{ab}}$ & $6.33 \pm 0.33^{\mathrm{bc}}$ & $4.33 \pm 0.33^{\text {de }}$ \\
\hline & $\mathrm{T}_{6}$ & $3.33 \pm 0.33^{\mathrm{abc}}$ & $5.00 \pm 0.00^{\mathrm{ab}}$ & $5.67 \pm 0.33^{\text {alacd }}$ & $4.67 \pm 0.33^{\mathrm{bc}}$ & $3.00 \pm 0.00^{2}$ & $4.33 \pm 0.33^{\mathrm{ab}}$ & $6.00 \pm 0.00^{\mathrm{cd}}$ & $4.33 \pm 0.33^{\mathrm{cdc}}$ \\
\hline & $\mathrm{T}_{7}$ & $3.00 \pm 0.00^{\mathrm{abc}}$ & $5.00 \pm 0.00^{\mathrm{ab}}$ & $5.33 \pm 0.67^{\mathrm{kcd}}$ & $4.33 \pm 0.33^{\mathrm{bc}}$ & $2.67 \pm 0.33^{a}$ & $4.00 \pm 0.00^{\mathrm{b}}$ & $5.67 \pm 0.33^{\mathrm{cdc}}$ & $4.00 \pm 0.00^{\mathrm{dc}}$ \\
\hline & $\mathrm{T}_{8}$ & $3.00 \pm 0.00^{\mathrm{abc}}$ & $5.00 \pm 0.00^{-\mathrm{bb}}$ & $5.33 \pm 0.33^{\text {bed }}$ & $4.00 \pm 0.00 \mathrm{c}$ & $2.67 \pm 0.33^{2}$ & $4.00 \pm 0.00^{\mathrm{bb}}$ & $5.33 \pm 0.33^{\text {def }}$ & $4.00 \pm 0.00^{\mathrm{de}}$ \\
\hline & $\mathrm{T}_{9}$ & $2.67 \pm 0.33^{\mathrm{bc}}$ & $4.33 \pm 0.33^{\mathrm{bc}}$ & $5.00 \pm 0.00^{\mathrm{cd}}$ & $4.00 \pm 0.00^{c}$ & $2.33 \pm 0.33^{a}$ & $4.00 \pm 0.00^{\mathrm{bb}}$ & $5.00 \pm 0.00^{\mathrm{cf}}$ & $3.67 \pm 0.33^{\text {cf }}$ \\
\hline & $\mathrm{T}_{10}$ & $2.33 \pm 0.33^{c}$ & $3.67 \pm 0.67^{\circ}$ & $4.33 \pm 0.67^{\mathrm{c}}$ & $3.67 \pm 0.33^{c}$ & $2.33 \pm 0.33^{a}$ & $3.33 \pm 0.33^{\mathrm{b}}$ & $4.67 \pm 0.33^{f}$ & $3.00 \pm 0.00^{f}$ \\
\hline \multirow{11}{*}{$\begin{array}{c}30 \mathrm{~cm} \\
\text { weed } \\
\text { distance }\end{array}$} & $\mathrm{T}_{0}$ & $4.00 \pm 0.00^{2}$ & $6.33 \pm 0.33^{a}$ & $7.33 \pm 0.33^{a}$ & $5.00 \pm 0.00^{\mathrm{a}}$ & $8.57 \pm 0.34^{2}$ & $3.67 \pm 0.33^{2}$ & $5.33 \pm 0.67^{\mathrm{ab}}$ & $8.00 \pm 0.58^{a}$ \\
\hline & $\mathrm{T}_{1}$ & $4.00 \pm 0.00^{2}$ & $5.67 \pm 0.33^{\mathrm{ab}}$ & $7.33 \pm 0.33^{2}$ & $4.33 \pm 0.33^{\mathrm{ab}}$ & $7.80 \pm 0.42^{\mathrm{ab}}$ & $3.67 \pm 0.33^{2}$ & $5.67 \pm 0.33^{\mathrm{a}}$ & $8.33 \pm 0.33^{a}$ \\
\hline & $\mathrm{T}_{2}$ & $3.67 \pm 0.33^{\mathrm{ab}}$ & $5.67 \pm 0.33^{\mathrm{ab}}$ & $6.67 \pm 0.33^{\mathrm{ab}}$ & $4.67 \pm 0.33^{\mathrm{ab}}$ & $7.17 \pm 0.09^{\mathrm{bc}}$ & $3.33 \pm 0.33^{\mathrm{ab}}$ & $5.33 \pm 0.33^{\mathrm{ab}}$ & $7.67 \pm 0.67^{\mathrm{ab}}$ \\
\hline & $\mathrm{T}_{3}$ & $4.00 \pm 0.00^{2}$ & $5.67 \pm 0.33^{\mathrm{ab}}$ & $6.33 \pm 0.33^{\text {alc }}$ & $4.67 \pm 0.33^{3 \mathrm{~b}}$ & $6.90 \pm 0.26^{\text {bdd }}$ & $3.33 \pm 0.33^{3 \mathrm{~b}}$ & $5.00 \pm 0.00^{\mathrm{abc}}$ & $7.67 \pm 0.33^{\mathrm{ab}}$ \\
\hline & $\mathrm{T}_{4}$ & $3.33 \pm 0.33^{3 \mathrm{bc}}$ & $4.67 \pm 0.33^{\mathrm{bc}}$ & $5.67 \pm 0.33^{\text {bcd }}$ & $4.33 \pm 0.33^{\mathrm{ab}}$ & $6.30 \pm 0.06^{\mathrm{cde}}$ & $3.33 \pm 0.33^{\mathrm{ab}}$ & $5.00 \pm 0.58^{8 \mathrm{abc}}$ & $6.33 \pm 0.88^{\mathrm{abc}}$ \\
\hline & $\mathrm{T}_{5}$ & $3.33 \pm 0.33^{\mathrm{abc}}$ & $4.33 \pm 0.33^{\mathrm{cd}}$ & $5.00 \pm 0.58^{\mathrm{de}}$ & $4.33 \pm 0.33^{\text {ab }}$ & $6.17 \pm 0.59^{\mathrm{dec}}$ & $3.00 \pm 0.00^{\mathrm{abc}}$ & $3.67 \pm 0.33^{c}$ & $5.67 \pm 0.88^{b c}$ \\
\hline & $\mathrm{T}_{6}$ & $3.00 \pm 0.00^{\mathrm{bc}}$ & $4.00 \pm 0.58^{\mathrm{cd}}$ & $4.67 \pm 0.33^{\text {ef }}$ & $4.33 \pm 0.33^{\mathrm{ab}}$ & $5.97 \pm 0.34^{\mathrm{dc}}$ & $3.00 \pm 0.00^{\mathrm{abc}}$ & $4.00 \pm 0.0 \mathrm{~b}^{\mathrm{bc}}$ & $5.67 \pm 0.33^{\mathrm{bc}}$ \\
\hline & $\mathrm{T}_{7}$ & $2.67 \pm 0.33^{c}$ & $4.67 \pm 0.33^{\mathrm{bc}}$ & $5.33 \pm 0.33^{\mathrm{cd}}$ & $4.00 \pm 0.00^{\mathrm{b}}$ & $5.77 \pm 0.32^{\mathrm{c}}$ & $3.00 \pm 0.00^{\mathrm{bc}}$ & $4.00 \pm 0.58^{\mathrm{bc}}$ & $5.67 \pm 0.88^{\mathrm{bc}}$ \\
\hline & $\mathrm{T}_{8}$ & $3.33 \pm 0.33^{\mathrm{abc}}$ & $3.33 \pm 0.33^{\mathrm{d}}$ & $4.00 \pm 0.00^{\mathrm{cf}}$ & $4.00 \pm 0.00^{\mathrm{b}}$ & $4.70 \pm 0.32^{\mathrm{f}}$ & $2.67 \pm 0.33^{\text {bc }}$ & $3.67 \pm 0.33^{c}$ & $5.00 \pm 0.58^{c}$ \\
\hline & $\mathrm{T}_{9}$ & $3.67 \pm 0.33^{3 \mathrm{~b}}$ & $3.67 \pm 0.33^{\mathrm{cd}}$ & $3.67 \pm 0.33^{f}$ & $4.00 \pm 0.00^{\mathrm{b}}$ & $4.63 \pm 0.20^{f}$ & $2.67 \pm 0.33^{\mathrm{bc}}$ & $4.00 \pm 0.58^{\mathrm{bc}}$ & $5.00 \pm 0.58^{c}$ \\
\hline & $\mathrm{T}_{10}$ & $3.33 \pm 0.33^{\mathrm{abc}}$ & $4.00 \pm 0.00^{\mathrm{cd}}$ & $3.67 \pm 0.33^{f}$ & $4.00 \pm 0.00^{\mathrm{b}}$ & $4.57 \pm 0.17^{\mathrm{f}}$ & $2.33 \pm 0.33^{c}$ & $3.67 \pm 0.33^{c}$ & $5.00 \pm 0.58^{c}$ \\
\hline \multirow{11}{*}{$\begin{array}{l}\text { Weed } \\
\text { density }\end{array}$} & $\mathrm{T}_{0}$ & $2.67 \pm 0.33^{2}$ & $6.00 \pm 0.00^{1}$ & $6.67 \pm 0.33^{a}$ & $5.67 \pm 0.33^{\mathrm{a}}$ & $3.67 \pm 0.33^{a}$ & $5.67 \pm 0.33^{2}$ & $8.00 \pm 0.58^{\mathrm{a}}$ & $6.33 \pm 0.88^{a}$ \\
\hline & $\mathrm{T}_{1}$ & $3.00 \pm 0.00^{2}$ & $5.33 \pm 0.33^{\text {ab }}$ & $6.00 \pm 0.00^{\mathrm{ab}}$ & $5.33 \pm 0.33^{\mathrm{ab}}$ & $3.33 \pm 0.33^{\mathrm{ab}}$ & $5.33 \pm 0.33^{2}$ & $8.33 \pm 0.33^{a}$ & $6.33 \pm 0.33^{a}$ \\
\hline & $\mathrm{T}_{2}$ & $3.00 \pm 0.00^{2}$ & $5.00 \pm 0.00^{\mathrm{blbc}}$ & $6.00 \pm 0.58^{\mathrm{bb}}$ & $5.33 \pm 0.33^{3 \mathrm{~b}}$ & $3.00 \pm 0.00^{\mathrm{sbc}}$ & $5.33 \pm 0.33^{\star}$ & $7.67 \pm 0.67^{\mathrm{ab}}$ & $5.67 \pm 0.67^{\mathrm{ab}}$ \\
\hline & $\mathrm{T}_{3}$ & $2.67 \pm 0.33^{a}$ & $4.00 \pm 0.58^{c}$ & $5.67 \pm 0.33^{\text {abc }}$ & $5.00 \pm 0.00^{\mathrm{ab}}$ & $3.00 \pm 0.00^{\text {the }}$ & $5.00 \pm 0.00^{\mathrm{bb}}$ & $7.67 \pm 0.33^{\mathrm{ab}}$ & $5.67 \pm 0.33^{\mathrm{ab}}$ \\
\hline & $\mathrm{T}_{4}$ & $2.33 \pm 0.33^{a}$ & $5.00 \pm 0.00^{\mathrm{bhc}}$ & $6.00 \pm 0.00^{\mathrm{ab}}$ & $4.67 \pm 0.33^{\mathrm{abc}}$ & $2.67 \pm 0.33^{\mathrm{bc}}$ & $4.33 \pm 0.33^{\mathrm{bc}}$ & $6.33 \pm 0.88^{\mathrm{abc}}$ & $4.67 \pm 0.33^{\text {bc }}$ \\
\hline & $T_{5}$ & $3.00 \pm 0.00^{2}$ & $4.33 \pm 0.33^{\mathrm{bc}}$ & $5.33 \pm 0.33^{\text {bad }}$ & $4.33 \pm 0.33^{\text {bed }}$ & $3.00 \pm 0.00^{\mathrm{bcc}}$ & $4.00 \pm 0.00^{\mathrm{dd}}$ & $5.67 \pm 0.88^{\mathrm{bc}}$ & $3.67 \pm 0.33^{\mathrm{bc}}$ \\
\hline & $\mathrm{T}_{6}$ & $2.33 \pm 0.33^{a}$ & $4.00 \pm 0.58^{c}$ & $5.33 \pm 0.33^{\text {bed }}$ & $4.33 \pm 0.33^{\text {bcd }}$ & $2.67 \pm 0.33^{b x}$ & $4.00 \pm 0.00^{c^{d}}$ & $5.67 \pm 0.33^{\mathrm{bc}}$ & $4.00 \pm 0.58^{b c}$ \\
\hline & $\mathrm{T}_{7}$ & $3.00 \pm 0.00^{2}$ & $4.33 \pm 0.33^{\mathrm{bc}}$ & $5.33 \pm 0.33^{\mathrm{bcd}}$ & $4.33 \pm 0.33^{\text {bed }}$ & $2.67 \pm 0.33^{\mathrm{bc}}$ & $3.67 \pm 0.33^{\mathrm{cd}}$ & $5.67 \pm 0.88^{\mathrm{bc}}$ & $4.00 \pm 0.58^{\text {bc }}$ \\
\hline & $\mathrm{T}_{8}$ & $3.00 \pm 0.00^{ \pm}$ & $4.67 \pm 0.33^{\mathrm{bc}}$ & $4.67 \pm 0.33^{\mathrm{cde}}$ & $3.67 \pm 0.33^{\text {cd }}$ & $2.33 \pm 0.33^{c}$ & $3.67 \pm 0.33^{\mathrm{cd}}$ & $5.00 \pm 0.5^{8 c}$ & $3.67 \pm 0.33^{c}$ \\
\hline & $\mathrm{T}_{9}$ & $2.67 \pm 0.33^{ \pm}$ & $4.00 \pm 0.00^{c}$ & $4.33 \pm 0.33^{\text {de }}$ & $3.67 \pm 0.33^{\mathrm{cd}}$ & $2.33 \pm 0.33^{c}$ & $3.67 \pm 0.33^{\mathrm{cd}}$ & $5.00 \pm 0.58^{c}$ & $3.67 \pm 0.67^{c}$ \\
\hline & $\mathrm{T}_{10}$ & $3.00 \pm 0.00^{2}$ & $4.67 \pm 0.33^{\mathrm{bc}}$ & $4.00 \pm 0.00^{c}$ & $3.33 \pm 0.33^{d}$ & $2.33 \pm 0.333^{c}$ & $3.33 \pm 0.33^{d}$ & $5.00 \pm 0.58^{c}$ & $3.33 \pm 0.67^{\mathrm{c}}$ \\
\hline
\end{tabular}

$\mathrm{T}_{2}=2$ weeds plant
${ }^{-1}, \mathrm{~T}_{3}=3$ weeds plant $^{-1}, \mathrm{~T}_{4}=4$ weeds plant 10 weed plant ${ }^{-1}$. 
Table 3. Effects of E. heterophylla weeds density and distance on the stem girth of $A$. esculentus (Var.'Kirikoi' and 'NHAe-40days')

\begin{tabular}{|c|c|c|c|c|c|c|c|c|c|}
\hline & \multirow{2}{*}{ Treatments } & \multicolumn{4}{|c|}{ Var. 'Kirikoi' } & \multicolumn{4}{|c|}{ Var. 'NHAe-40days' } \\
\hline & & 2 WAP & $4 \mathrm{WAP}$ & $6 \mathrm{WAP}$ & $8 \mathrm{WAP}$ & 2 WAP & $4 \mathrm{WAP}$ & $6 \mathrm{WAP}$ & $8 \mathrm{WAP}$ \\
\hline \multirow{11}{*}{$\begin{array}{c}20 \mathrm{~cm} \\
\text { weed } \\
\text { distance }\end{array}$} & $\mathrm{T}_{0}$ & $0.46 \pm 0.03^{2}$ & $0.80 \pm 0.02^{\mathrm{a}}$ & $1.60 \pm 0.06^{a}$ & $1.93 \pm 0.03^{a}$ & $0.43 \pm 0.07^{\mathrm{a}}$ & $1.40 \pm 0.10^{2}$ & $1.70 \pm 0.15^{2}$ & $2.20 \pm 0.2 \mathrm{l}^{2}$ \\
\hline & $\mathrm{T}_{1}$ & $0.44 \pm 0.03^{\mathrm{ab}}$ & $0.73 \pm 0.03^{\mathrm{ab}}$ & $1.41 \pm 0.10^{\text {tb }}$ & $1.90 \pm 0.00^{2}$ & $0.40 \pm 0.06^{\mathrm{ab}}$ & $1.20 \pm 0.06^{\text {th }}$ & $1.47 \pm 0.09^{\mathrm{ab}}$ & $1.90 \pm 0.10^{\text {ab }}$ \\
\hline & $\mathrm{T}_{2}$ & $0.37 \pm 0.03^{\mathrm{bc}}$ & $0.67 \pm 0.03^{\mathrm{bc}}$ & $1.33 \pm 0.15^{\mathrm{ab}}$ & $1.90 \pm 0.00^{\mathrm{a}}$ & $0.33 \pm 0.03^{\text {abc }}$ & $1.03 \pm 0.09^{\mathrm{bc}}$ & $1.27 \pm 0.12^{\mathrm{bc}}$ & $1.63 \pm 0.15^{\mathrm{bc}}$ \\
\hline & $\mathrm{T}_{3}$ & $0.37 \pm 0.07^{\mathrm{zbc}}$ & $0.63 \pm 0.03^{c}$ & $1.37 \pm 0.12^{\mathrm{ab}}$ & $1.90 \pm 0.00^{2}$ & $0.33 \pm 0.03^{\text {bic }}$ & $1.00 \pm 0.06^{\mathrm{bc}}$ & $1.20 \pm 0.06^{\mathrm{bc}}$ & $1.57 \pm 0.09^{\mathrm{kc}}$ \\
\hline & $\mathrm{T}_{4}$ & $0.35 \pm 0.03^{\text {abc }}$ & $0.60 \pm 0.00^{\text {cd }}$ & $1.43 \pm 0.02^{\mathrm{ab}}$ & $1.90 \pm 0.00^{\mathrm{a}}$ & $0.33 \pm 0.03^{\mathrm{abc}}$ & $1.03 \pm 0.09^{\mathrm{bc}}$ & $1.27 \pm 0.12^{\mathrm{bc}}$ & $1.63 \pm 0.15^{b c}$ \\
\hline & $\mathrm{T}_{5}$ & $0.33 \pm 0.03^{\mathrm{bc}}$ & $0.53 \pm 0.03^{\text {de }}$ & $1.43 \pm 0.03^{\mathrm{ab}}$ & $1.90 \pm 0.00 \mathrm{a}$ & $0.33 \pm 0.03^{\text {abc }}$ & $0.97 \pm 0.07^{\mathrm{bc}}$ & $1.17 \pm 0.07^{\mathrm{bcd}}$ & $1.50 \pm 0.10^{\mathrm{bc}}$ \\
\hline & $\mathrm{T}_{6}$ & $0.27 \pm 0.03^{c}$ & $0.50 \pm 0.00^{c}$ & $1.40 \pm 0.06^{\mathrm{ab}}$ & $1.90 \pm 0.00^{\mathrm{a}}$ & $0.30 \pm 0.00^{\mathrm{bc}}$ & $0.97 \pm 0.03^{\mathrm{bc}}$ & $1.17 \pm 0.03^{\text {bcd }}$ & $1.53 \pm 0.07^{\mathrm{bc}}$ \\
\hline & $\mathrm{T}_{7}$ & $0.27 \pm 0.03^{c}$ & $0.53 \pm 0.03^{\mathrm{de}}$ & $1.33 \pm 0.09^{\mathrm{ab}}$ & $1.90 \pm 0.00 \mathrm{a}$ & $0.30 \pm 0.00^{\mathrm{bc}}$ & $0.90 \pm 0.00^{\mathrm{cd}}$ & $1.10 \pm 0.00^{\text {cd }}$ & $1.40 \pm 0.00^{\text {cd }}$ \\
\hline & $\mathrm{T}_{8}$ & $0.27 \pm 0.03^{c}$ & $0.53 \pm 0.03^{\text {de }}$ & $1.20 \pm 0.15^{\mathrm{b}}$ & $1.90 \pm 0.00 \mathrm{a}$ & $0.27 \pm 0.33^{c}$ & $0.83 \pm 0.07^{\mathrm{dd}}$ & $1.03 \pm 0.07^{\mathrm{cd}}$ & $1.30 \pm 0.10^{\text {cd }}$ \\
\hline & $\mathrm{T}_{9}$ & $0.30 \pm 0.00^{c}$ & $0.47 \pm 0.03^{\mathrm{c}}$ & $1.21 \pm 0.06^{\mathrm{b}}$ & $1.90 \pm 0.00^{\mathrm{a}}$ & $0.27 \pm 0.03^{c}$ & $0.80 \pm 0.10^{\text {cd }}$ & $0.97 \pm 0.13^{\text {cd }}$ & $1.23 \pm 0.17^{\mathrm{cd}}$ \\
\hline & $\mathrm{T}_{10}$ & $0.33 \pm 0.03^{\mathrm{bc}}$ & $0.47 \pm 0.03^{c}$ & $1.27 \pm 0.15^{\mathrm{ab}}$ & $1.90 \pm 0.00^{2}$ & $0.23 \pm 0.03^{c}$ & $0.70 \pm 0.06^{\mathrm{d}}$ & $0.87 \pm 0.09^{d}$ & $1.07 \pm 0.09^{d}$ \\
\hline \multirow{11}{*}{$\begin{array}{c}30 \mathrm{~cm} \\
\text { weed } \\
\text { distance }\end{array}$} & $\mathrm{T}_{0}$ & $0.40 \pm 0.06^{\mathrm{a}}$ & $1.47 \pm 0.09^{\mathrm{a}}$ & $3.60 \pm 0.12^{\mathrm{a}}$ & $1.90 \pm 0.00^{\mathrm{a}}$ & $0.47 \pm 0.03^{a}$ & $1.43 \pm 0.12^{2}$ & $2.23 \pm 0.35^{a}$ & $2.23 \pm 0.23^{a}$ \\
\hline & $\mathrm{T}_{1}$ & $0.40 \pm 0.00^{1}$ & $1.03 \pm 0.09^{\mathrm{b}}$ & $1.63 \pm 0.09^{b}$ & $1.90 \pm 0.00^{\mathrm{a}}$ & $0.40 \pm 0.06^{\mathrm{ab}}$ & $1.20 \pm 0.12^{\mathrm{b}}$ & $1.87 \pm 0.15^{\mathrm{ab}}$ & $1.90 \pm 0.17^{\mathrm{ab}}$ \\
\hline & $\mathrm{T}_{2}$ & $0.33 \pm 0.03^{\mathrm{ab}}$ & $0.97 \pm 0.09^{\mathrm{bc}}$ & $1.50 \pm 0.06^{\mathrm{bc}}$ & $1.90 \pm 0.00 \mathrm{a}$ & $0.40 \pm 0.00^{\mathrm{ab}}$ & $1.10 \pm 0.06^{\mathrm{bc}}$ & $1.63 \pm 0.15^{\mathrm{bc}}$ & $1.70 \pm 0.12^{\mathrm{bc}}$ \\
\hline & $\mathrm{T}_{3}$ & $0.33 \pm 0.03^{3^{\mathrm{b}}}$ & $1.03 \pm 0.09^{b}$ & $1.47 \pm 0.26^{b c}$ & $1.90 \pm 0.00^{\mathrm{a}}$ & $0.33 \pm 0.03^{\mathrm{bc}}$ & $1.00 \pm 0.00^{\mathrm{bcd}}$ & $1.53 \pm 0.07^{\mathrm{bcd}}$ & $1.57 \pm 0.03^{\text {bcd }}$ \\
\hline & $\mathrm{T}_{4}$ & $0.33 \pm 0.03^{\mathrm{ab}}$ & $0.97 \pm 0.12^{\mathrm{bc}}$ & $1.30 \pm 0.10^{\mathrm{bcd}}$ & $1.90 \pm 0.00^{\mathrm{a}}$ & $0.30 \pm 0.06^{\mathrm{bc}}$ & $1.00 \pm 0.06^{\text {bcd }}$ & $1.63 \pm 0.15^{\mathrm{bc}}$ & $1.60 \pm 0.10^{\text {bcd }}$ \\
\hline & $\mathrm{T}_{5}$ & $0.33 \pm 0.03^{\mathrm{ab}}$ & $0.83 \pm 0.03^{\mathrm{bc}}$ & $1.27 \pm 0.12^{\mathrm{bcd}}$ & $1.90 \pm 0.00^{2}$ & $0.33 \pm 0.03^{b c}$ & $0.97 \pm 0.03^{\text {cd }}$ & $1.47 \pm 0.07^{\mathrm{bcd}}$ & $1.50 \pm 0.06^{\mathrm{cd}}$ \\
\hline & $\mathrm{T}_{6}$ & $0.33 \pm 0.03^{\mathrm{ab}}$ & $0.77 \pm 0.03^{\mathrm{cd}}$ & $1.23 \pm 0.13^{\mathrm{cd}}$ & $1.90 \pm 0.00^{\mathrm{a}}$ & $0.33 \pm 0.03^{b c}$ & $1.00 \pm 0.06^{\mathrm{bcd}}$ & $1.53 \pm 0.07^{\mathrm{bcd}}$ & $1.57 \pm 0.09^{\mathrm{bcd}}$ \\
\hline & $\mathrm{T}_{7}$ & $0.30 \pm 0.00^{\mathrm{ab}}$ & $0.77 \pm 0.03^{\text {dd }}$ & $1.07 \pm 0.07^{\mathrm{d}}$ & $1.90 \pm 0.00^{\mathrm{a}}$ & $0.30 \pm 0.00^{\mathrm{bc}}$ & $0.90 \pm 0.00^{\text {cde }}$ & $1.40 \pm 0.00^{\mathrm{bcd}}$ & $1.40 \pm 0.00^{\mathrm{cdc}}$ \\
\hline & $\mathrm{T}_{8}$ & $0.30 \pm 0.00^{\mathrm{bb}}$ & $0.57 \pm 0.03^{\text {de }}$ & $1.07 \pm 0.07^{\mathrm{d}}$ & $1.90 \pm 0.00^{\mathrm{a}}$ & $0.27 \pm 0.03^{c}$ & $0.83 \pm 0.03^{\text {de }}$ & $1.33 \pm 0.07^{\mathrm{cd}}$ & $1.30 \pm 0.06^{\mathrm{dc}}$ \\
\hline & $\mathrm{T}_{9}$ & $0.27 \pm 0.03^{b}$ & $0.53 \pm 0.03^{\mathrm{c}}$ & $1.03 \pm 0.03^{\mathrm{d}}$ & $1.90 \pm 0.00 \mathrm{a}$ & $0.27 \pm 0.03^{c}$ & $0.80 \pm 0.06^{\mathrm{dc}}$ & $1.23 \pm 0.17^{\mathrm{dd}}$ & $1.23 \pm 0.12^{\mathrm{dc}}$ \\
\hline & $\mathrm{T}_{10}$ & $0.27 \pm 0.03^{\mathrm{b}}$ & $0.50 \pm 0.05 \mathrm{e}$ & $1.07 \pm 0.03^{d}$ & $1.90 \pm 0.00^{2}$ & $0.23 \pm 0.03^{c}$ & $0.70 \pm 0.06 \mathrm{e}$ & $1.10 \pm 0.10^{\mathrm{d}}$ & $1.07 \pm 0.09^{c}$ \\
\hline \multirow{11}{*}{$\begin{array}{l}\text { Weed } \\
\text { density }\end{array}$} & $\mathrm{T}_{0}$ & $0.50 \pm 0.00^{2}$ & $0.83 \pm 0.09 \mathrm{a}$ & $1.60 \pm 0.20^{\mathrm{ab}}$ & $1.90 \pm 0.00^{2}$ & $0.47 \pm 0.09^{a}$ & $1.93 \pm 0.29^{\circ}$ & $2.23 \pm 0.35^{2}$ & $2.73 \pm 0.41^{a}$ \\
\hline & $\mathrm{T}_{1}$ & $0.37 \pm 0.03^{ \pm}$ & $0.77 \pm 0.03^{\mathrm{ab}}$ & $1.67 \pm 0.12^{\mathrm{a}}$ & $1.81 \pm 0.03^{\text {b }}$ & $0.47 \pm 0.03^{a}$ & $1.60 \pm 0.12^{\text {ab }}$ & $1.87 \pm 0.15^{\mathrm{ab}}$ & $2.30 \pm 0.17^{\mathrm{ab}}$ \\
\hline & $\mathrm{T}_{2}$ & $0.37 \pm 0.03^{2}$ & $0.70 \pm 0.06^{\text {ble }}$ & $1.57 \pm 0.12^{\mathrm{atc}}$ & $1.77 \pm 0.01^{\text {bb }}$ & $0.40 \pm 0.00^{\text {ab }}$ & $1.40 \pm 0.12^{\mathrm{bc}}$ & $1.63 \pm 0.15^{\mathrm{bc}}$ & $2.00 \pm 0.17^{\mathrm{kc}}$ \\
\hline & $\mathrm{T}_{3}$ & $0.37 \pm 0.03^{2}$ & $0.67 \pm 0.07^{\mathrm{bod}}$ & $1.33 \pm 0.17^{\text {alacd }}$ & $1.58 \pm 0.05^{\mathrm{cd}}$ & $0.37 \pm 0.03^{\mathrm{ab}}$ & $1.33 \pm 0.07^{\mathrm{bcd}}$ & $1.53 \pm 0.07^{\mathrm{bcd}}$ & $1.90 \pm 0.10^{b c}$ \\
\hline & $\mathrm{T}_{4}$ & $0.33 \pm 0.03^{\mathrm{bc}}$ & $0.63 \pm 0.03^{\text {bede }}$ & $1.30 \pm 0.10^{\text {bcd }}$ & $1.71 \pm 0.02^{\mathrm{bc}}$ & $0.37 \pm 0.03^{\mathrm{ab}}$ & $1.40 \pm 0.12^{\mathrm{bc}}$ & $1.63 \pm 0.15^{\mathrm{bc}}$ & $2.00 \pm 0.17^{b c}$ \\
\hline & $\mathrm{T}_{5}$ & $0.30 \pm 0.00^{\text {bed }}$ & $0.53 \pm 0.03^{\mathrm{de}}$ & $1.07 \pm 0.07^{\mathrm{d}}$ & $1.74 \pm 0.13^{b}$ & $0.33 \pm 0.03^{b c}$ & $1.27 \pm 0.07^{\mathrm{bcd}}$ & $1.47 \pm 0.07^{\mathrm{bcd}}$ & $1.80 \pm 0.10^{\text {bcd }}$ \\
\hline & $\mathrm{T}_{6}$ & $0.30 \pm 0.00^{\text {bcd }}$ & $0.60 \pm 0.06^{\mathrm{dc}}$ & $1.23 \pm 0.07^{\mathrm{cd}}$ & $1.51 \pm 0.01^{\mathrm{d}}$ & $0.33 \pm 0.03^{\mathrm{bc}}$ & $1.33 \pm 0.07^{\mathrm{bcd}}$ & $1.53 \pm 0.07^{\mathrm{bcd}}$ & $1.90 \pm 0.10^{b x}$ \\
\hline & $\mathrm{T}_{7}$ & $0.27 \pm 0.03^{\text {cde }}$ & $0.57 \pm 0.03^{\text {cde }}$ & $1.13 \pm 0.09^{d}$ & $1.55 \pm 0.01^{\mathrm{d}}$ & $0.30 \pm 0.00^{\text {bcd }}$ & $1.20 \pm 0.00^{\mathrm{bcd}}$ & $1.40 \pm 0.00^{\text {bcd }}$ & $1.70 \pm 0.00^{\text {cd }}$ \\
\hline & $\mathrm{T}_{8}$ & $0.27 \pm 0.03^{\text {cdc }}$ & $0.53 \pm 0.03^{\text {de }}$ & $1.10 \pm 0.06^{\mathrm{d}}$ & $1.56 \pm 0.01^{\mathrm{d}}$ & $0.30 \pm 0.00^{\text {bed }}$ & $1.13 \pm 0.07^{\mathrm{cd}}$ & $1.33 \pm 0.07^{\mathrm{cd}}$ & $1.60 \pm 0.10^{\text {cd }}$ \\
\hline & $\mathrm{T}_{9}$ & $0.23 \pm 0.03^{\text {de }}$ & $0.53 \pm 0.03^{\text {de }}$ & $1.03 \pm 0.03^{\mathrm{d}}$ & $1.51 \pm 0.02^{\mathrm{d}}$ & $0.23 \pm 0.03^{\text {dd }}$ & $1.07 \pm 0.13^{\mathrm{dd}}$ & $1.23 \pm 0.17^{\mathrm{dd}}$ & $1.50 \pm 0.20^{\mathrm{cd}}$ \\
\hline & $\mathrm{T}_{10}$ & $0.20 \pm 0.00^{c}$ & $0.50 \pm 0.00^{c}$ & $1.00 \pm 0.06^{d}$ & $1.47 \pm 0.02^{\mathrm{d}}$ & $0.20 \pm 0.00^{d}$ & $0.93 \pm 0.07^{\mathrm{d}}$ & $1.10 \pm 0.10^{d}$ & $1.30 \pm 0.10^{d}$ \\
\hline
\end{tabular}

Values represent Means \pm SEM, $n=3$, values with same superscript along the column are significantly the same at $p<0.05 ; T_{0}=c_{0}$ trol, $T_{1}=1$ weed, $T_{1}=1$ weed plant ${ }^{-1}$,

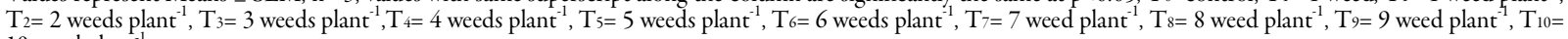
10 weed plant ${ }^{-1}$

Table 4. Effects of E. heterophylla weeds density and distance on the leaves area $\left(\mathrm{cm}^{2}\right)$ of $A$. esculentus (Var.'Kirikoi' and 'NHAe-40days')

\begin{tabular}{|c|c|c|c|c|c|c|c|c|c|}
\hline & \multirow{2}{*}{ Treatments } & \multicolumn{4}{|c|}{ Var. 'Kirikoi' } & \multicolumn{4}{|c|}{ Var. 'NHAe-40days' } \\
\hline & & $2 \mathrm{WAP}$ & $4 \mathrm{WAP}$ & $6 \mathrm{WAP}$ & $8 \mathrm{WAP}$ & $2 \mathrm{WAP}$ & $4 \mathrm{WAP}$ & $6 \mathrm{WAP}$ & $8 \mathrm{WAP}$ \\
\hline \multirow{11}{*}{$\begin{array}{c}20 \mathrm{~cm} \\
\text { weed } \\
\text { distance }\end{array}$} & $\mathrm{T}_{0}$ & $16.25 \pm 0.40^{2}$ & $31.58 \pm 1.09^{2}$ & $45.80 \pm 1.61^{4}$ & $39.76 \pm 2.53^{4}$ & $13.15 \pm 0.55^{a}$ & $24.88 \pm 0.74^{2}$ & $48.34 \pm 1.51^{2}$ & $36.61 \pm 1.10^{2}$ \\
\hline & $\mathrm{T}_{1}$ & $13.68 \pm 0.23^{b}$ & $30.36 \pm 1.03^{\text {ab }}$ & $44.49 \pm 0.50^{2}$ & $40.20 \pm 0.23^{a}$ & $13.14 \pm 1.11^{2}$ & $24.52 \pm 1.11^{2}$ & $47.26 \pm 1.34^{2}$ & $35.89 \pm 1.19^{4}$ \\
\hline & $\mathrm{T}_{2}$ & $13.41 \pm 0.39^{\mathrm{bc}}$ & $28.21 \pm 1.21^{\mathrm{bc}}$ & $45.67 \pm 1.06^{a}$ & $38.48 \pm 1.50^{0^{\mathrm{bb}}}$ & $11.98 \pm 0.97^{\mathrm{ab}}$ & $22.57 \pm 1.10^{2 \mathrm{~b}}$ & $43.77 \pm 1.48^{\mathrm{ab}}$ & $33.17 \pm 1.28^{\mathrm{ab}}$ \\
\hline & $\mathrm{T}_{3}$ & $12.46 \pm 0.32^{c}$ & $27.54 \pm 0.91^{\mathrm{bc}}$ & $42.15 \pm 1.57^{\mathrm{ab}}$ & $38.08 \pm 0.78^{\mathrm{ab}}$ & $10.80 \pm 0.65^{\text {bc }}$ & $20.52 \pm 0.91^{\mathrm{bc}}$ & $39.96 \pm 1.45^{\mathrm{bc}}$ & $30.24 \pm 1.18^{\mathrm{bc}}$ \\
\hline & $\mathrm{T}_{4}$ & $9.71 \pm 0.01^{\mathrm{c}}$ & $25.92 \pm 0.64^{\mathrm{cd}}$ & $39.84 \pm 1.99^{\circ}$ & $37.78 \pm 3.40^{\mathrm{sb}}$ & $10.16 \pm 0.58^{\mathrm{bdd}}$ & $20.82 \pm 1.37^{\mathrm{bc}}$ & $42.14 \pm 3.17^{\mathrm{b}}$ & $31.48 \pm 2.26^{\mathrm{b}}$ \\
\hline & $\mathrm{T}_{5}$ & $9.18 \pm 0.06^{\mathrm{d}}$ & $23.07 \pm 1.05^{\mathrm{d}}$ & $34.91 \pm 0.44^{c}$ & $35.97 \pm 0.94^{\mathrm{ab}}$ & $9.02 \pm 0.73^{\mathrm{cde}}$ & $18.17 \pm 1.10^{\text {cd }}$ & $36.47 \pm 2.37^{\mathrm{cd}}$ & $27.32 \pm 1.70^{c \mathrm{~d}}$ \\
\hline & $\mathrm{T}_{6}$ & $6.56 \pm 0.42^{c}$ & $19.83 \pm 0.78^{\mathrm{c}}$ & $33.10 \pm 1.62^{\mathrm{cd}}$ & $33.82 \pm 1.04^{\mathrm{bc}}$ & $8.68 \pm 0.56^{\text {cdef }}$ & $17.24 \pm 0.7 \mathrm{I}^{\mathrm{de}}$ & $34.35 \pm 1.04^{\mathrm{dc}}$ & $25.80 \pm 0.88^{d c}$ \\
\hline & $\mathrm{T}_{7}$ & $3.64 \pm 0.29^{f}$ & $19.67 \pm 0.55^{\circ}$ & $30.39 \pm 1.62^{\mathrm{de}}$ & $29.38 \pm 1.34^{\text {cd }}$ & $8.42 \pm 0.37^{\text {def }}$ & $16.17 \pm 0.47^{\text {def }}$ & $31.67 \pm 0.97^{\mathrm{d} f f}$ & $23.92 \pm 0.70^{\text {def }}$ \\
\hline & $\mathrm{T}_{8}$ & $3.96 \pm 0.40^{f}$ & $16.11 \pm 0.98^{f}$ & $26.59 \pm 1.53^{c}$ & $25.05 \pm 0.99^{d e}$ & $7.77 \pm 0.87^{\text {cf }}$ & $15.14 \pm 0.96^{\mathrm{cgg}}$ & $29.90 \pm 1.14^{\mathrm{ffg}}$ & $22.52 \pm 1.05^{\mathrm{fg}_{g}}$ \\
\hline & $\mathrm{T}_{9}$ & $4.17 \pm 0.35^{f}$ & $15.58 \pm 1.09^{f}$ & $22.30 \pm 1.64^{\mathrm{f}}$ & $24.44 \pm 1.39^{\circ}$ & $6.66 \pm 0.29^{f}$ & $13.51 \pm 0.36^{\mathrm{g}}$ & $27.21 \pm 0.51^{\text {fg }}$ & $20.36 \pm 0.43^{5}$ \\
\hline & $T_{10}$ & $4.55 \pm 0.41^{f}$ & $13.35 \pm 1.24^{f}$ & $20.75 \pm 0.36^{t}$ & $19.17 \pm 0.66^{\mathrm{f}}$ & $6.53 \pm 0.11^{f}$ & $12.69 \pm 0.03 \mathrm{~s}$ & $25.03 \pm 0.16^{8}$ & $18.86 \pm 0.07 \mathrm{~s}$ \\
\hline \multirow{11}{*}{$\begin{array}{c}30 \mathrm{~cm} \\
\text { weed } \\
\text { distance }\end{array}$} & $\mathrm{T}_{0}$ & $14.75 \pm 1.59^{2}$ & $28.20 \pm 1.35^{a}$ & $42.95 \pm 2.54^{a}$ & $38.59 \pm 1.50^{2}$ & $16.28 \pm 0.67^{\mathrm{ab}}$ & $28.01 \pm 1.06^{a}$ & $51.47 \pm 1.92^{\mathrm{a}}$ & $39.74 \pm 1.48^{a}$ \\
\hline & $\mathrm{T}_{1}$ & $12.47 \pm 0.40^{\mathrm{b}}$ & $28.22 \pm 1.37^{\mathrm{a}}$ & $41.64 \pm 3.14^{\mathrm{a}}$ & $35.63 \pm 2.31^{\mathrm{ab}}$ & $16.96 \pm 0.39^{\mathrm{a}}$ & $28.33 \pm 0.52^{a}$ & $51.08 \pm 1.04^{a}$ & $39.70 \pm 0.76^{a}$ \\
\hline & $\mathrm{T}_{2}$ & $8.72 \pm 0.47^{\mathrm{c}}$ & $26.79 \pm 0.37^{a}$ & $40.72 \pm 1.26^{\mathrm{ab}}$ & $38.77 \pm 1.01^{a}$ & $16.14 \pm 0.42^{2 \mathrm{~b}}$ & $26.74 \pm 0.47^{\mathrm{ab}}$ & $47.93 \pm 0.88^{\mathrm{ab}}$ & $37.33 \pm 0.65^{\text {ab }}$ \\
\hline & $\mathrm{T}_{3}$ & $8.45 \pm 1.04^{\mathrm{cd}}$ & $26.99 \pm 0.58^{a}$ & $39.10 \pm 1.04^{\mathrm{ab}}$ & $35.66 \pm 1.59^{\mathrm{ab}}$ & $15.38 \pm 0.29^{\mathrm{ab}}$ & $25.10 \pm 0.43^{\mathrm{ab}}$ & $44.54 \pm 0.91^{\mathrm{b}}$ & $34.82 \pm 0.66^{\mathrm{b}}$ \\
\hline & $\mathrm{T}_{4}$ & $7.74 \pm 0.22^{\mathrm{cde}}$ & $25.77 \pm 1.79^{\mathrm{ab}}$ & $38.00 \pm 2.17^{\mathrm{ab}}$ & $30.76 \pm 1.56^{\mathrm{bc}}$ & $13.70 \pm 0.51^{\mathrm{bc}}$ & $24.36 \pm 0.48^{\mathrm{b}}$ & $45.68 \pm 2.29^{b}$ & $35.02 \pm 1.38^{\mathrm{b}}$ \\
\hline & $\mathrm{T}_{5}$ & $8.24 \pm 0.57^{\mathrm{cd}}$ & $27.18 \pm 1.40^{2}$ & $38.39 \pm 1.32^{\mathrm{ab}}$ & $33.72 \pm 2.14^{\mathrm{blc}}$ & $11.55 \pm 0.6^{4 c}$ & $20.70 \pm 0.71^{c}$ & $39.00 \pm 1.92^{c}$ & $29.85 \pm 1.27^{c}$ \\
\hline & $\mathrm{T}_{6}$ & $7.25 \pm 0.31^{\mathrm{cde}}$ & $21.97 \pm 0.66^{\mathrm{cd}}$ & $37.40 \pm 0.57^{\mathrm{ab}}$ & $30.72 \pm 0.74^{\mathrm{bc}}$ & $11.64 \pm 1.87^{c}$ & $20.20 \pm 2.05^{c}$ & $37.31 \pm 2.39^{\mathrm{cd}}$ & $28.75 \pm 2.22^{c}$ \\
\hline & $\mathrm{T}_{7}$ & $6.20 \pm 0.55^{\text {de }}$ & $22.56 \pm 1.59^{\mathrm{bc}}$ & $38.73 \pm 1.03^{\mathrm{ab}}$ & $32.99 \pm 2.39^{\mathrm{bc}}$ & $11.52 \pm 0.87^{c}$ & $19.27 \pm 1.12^{c}$ & $34.77 \pm 1.68^{\mathrm{cde}}$ & $27.02 \pm 1.39^{\mathrm{cd}}$ \\
\hline & $\mathrm{T}_{8}$ & $5.92 \pm 0.45^{c}$ & $21.29 \pm 1.21^{\mathrm{cd}}$ & $36.65 \pm 2.34^{\mathrm{ab}}$ & $31.91 \pm 1.80^{\mathrm{bc}}$ & $11.34 \pm 1.36^{c}$ & $18.72 \pm 1.41^{\mathrm{c}}$ & $33.47 \pm 1.53^{\mathrm{dc}}$ & $26.10 \pm 1.47^{\mathrm{cd}}$ \\
\hline & $\mathrm{T}_{9}$ & $5.78 \pm 0.49^{c}$ & $18.80 \pm 0.39^{\circ}$ & $36.66 \pm 1.48^{\mathrm{ab}}$ & $29.66 \pm 1.06^{c}$ & $10.74 \pm 1.41^{\mathrm{cd}}$ & $17.59 \pm 1.50^{c}$ & $31.29 \pm 1.67^{\mathrm{cf}}$ & $24.44 \pm 1.59^{\mathrm{d}}$ \\
\hline & $\mathrm{T}_{10}$ & $3.26 \pm 0.46^{6}$ & $17.54 \pm 0.65^{\circ}$ & $34.85 \pm 2.28^{b}$ & $30.01 \pm 1.72 b^{c}$ & $8.15 \pm 0.62^{\mathrm{d}}$ & $14.31 \pm 0.59^{\mathrm{d}}$ & $26.65 \pm 0.55^{f}$ & $20.48 \pm 0.56^{c}$ \\
\hline \multirow{11}{*}{$\begin{array}{l}\text { Weed } \\
\text { density }\end{array}$} & $\mathrm{T}_{0}$ & $10.13 \pm 0.25^{2}$ & $22.94 \pm 0.44^{2}$ & $36.86 \pm 1.71^{a}$ & $33.43 \pm 0.92^{\mathrm{a}}$ & $13.34 \pm 0.73^{\mathrm{a}}$ & $25.07 \pm 0.99^{2}$ & $48.53 \pm 1.76^{2}$ & $36.80 \pm 1.35^{a}$ \\
\hline & $\mathrm{T}_{1}$ & $8.77 \pm 0.66^{\mathrm{ab}}$ & $22.45 \pm 0.91^{\mathrm{ab}}$ & $35.08 \pm 1.40^{\mathrm{ab}}$ & $32.44 \pm 1.23^{\mathrm{ab}}$ & $12.83 \pm 0.50^{0 \mathrm{~b}}$ & $24.20 \pm 0.30^{2}$ & $46.95 \pm 0.57^{a}$ & $35.57 \pm 0.34^{a}$ \\
\hline & $\mathrm{T}_{2}$ & $8.81 \pm 0.60^{\mathrm{ab}}$ & $21.49 \pm 1.60^{\mathrm{ab}}$ & $34.78 \pm 0.91^{\mathrm{ab}}$ & $31.13 \pm 0.69^{9 b c}$ & $10.52 \pm 0.94^{\mathrm{cd}}$ & $21.12 \pm 0.71^{\mathrm{b}}$ & $42.31 \pm 0.44^{\mathrm{b}}$ & $31.72 \pm 0.53^{\mathrm{b}}$ \\
\hline & $\mathrm{T}_{3}$ & $8.28 \pm 0.59^{\circ}$ & $21.60 \pm 1.48^{\mathrm{ab}}$ & $34.25 \pm 1.55^{\mathrm{ab}}$ & $30.23 \pm 1.13^{\text {abc }}$ & $11.06 \pm 0.68^{\mathrm{bc}}$ & $20.78 \pm 0.65^{b}$ & $40.22 \pm 0.88^{b}$ & $30.50 \pm 0.72^{\mathrm{b}}$ \\
\hline & $\mathrm{T}_{4}$ & $6.03 \pm 0.45^{\circ}$ & $21.17 \pm 0.40^{\mathrm{ab}}$ & $34.32 \pm 1.64^{\mathrm{bb}}$ & $29.14 \pm 1.24^{\text {bad }}$ & $10.80 \pm 0.54^{c}$ & $21.46 \pm 0.74^{b}$ & $42.78 \pm 2.47^{\mathrm{b}}$ & $32.12 \pm 1.58^{b}$ \\
\hline & $\mathrm{T}_{5}$ & $5.41 \pm 0.41^{\mathrm{cd}}$ & $19.93 \pm 0.74^{\mathrm{bc}}$ & $32.28 \pm 0.85^{\mathrm{bc}}$ & $29.24 \pm 1.10^{\mathrm{bcd}}$ & $8.20 \pm 0.70^{c}$ & $17.35 \pm 1.36^{c}$ & $35.65 \pm 2.75^{c}$ & $26.50 \pm 2.05^{c}$ \\
\hline & $\mathrm{T}_{6}$ & $5.28 \pm 0.74^{\mathrm{cd}}$ & $19.83 \pm 0.68^{\mathrm{bc}}$ & $29.75 \pm 1.35^{c}$ & $28.50 \pm 1.62^{\text {cd }}$ & $8.81 \pm 0.33^{\mathrm{de}}$ & $17.36 \pm 0.45^{c}$ & $34.48 \pm 0.75^{c}$ & $25.92 \pm 0.60^{c}$ \\
\hline & $\mathrm{T}_{7}$ & $5.01 \pm 0.72^{\mathrm{cd}}$ & $17.55 \pm 1.17^{\mathrm{bc}}$ & $28.99 \pm 0.42^{\mathrm{cd}}$ & $26.30 \pm 0.42^{\mathrm{d}}$ & $8.49 \pm 0.73^{\mathrm{e}}$ & $16.24 \pm 1.03^{\mathrm{cd}}$ & $31.74 \pm 1.64^{\text {cd }}$ & $23.99 \pm 1.33^{\text {cd }}$ \\
\hline & $\mathrm{T}_{8}$ & $5.25 \pm 0.20^{\mathrm{cd}}$ & $17.49 \pm 0.40^{\mathrm{bc}}$ & $25.88 \pm 0.80^{\mathrm{de}}$ & $20.94 \pm 1.38^{c}$ & $6.80 \pm 0.73^{c}$ & $14.18 \pm 0.65^{\mathrm{de}}$ & $28.93 \pm 0.53^{\mathrm{dc}}$ & $21.55 \pm 0.59^{\mathrm{de}}$ \\
\hline & $\mathrm{T}_{9}$ & $4.22 \pm 0.12^{\mathrm{d}}$ & $17.64 \pm 0.54^{\mathrm{bc}}$ & $25.46 \pm 1.05^{\mathrm{dc}}$ & $20.81 \pm 1.21^{\mathrm{c}}$ & $7.08 \pm 0.06^{\mathrm{e}}$ & $13.93 \pm 0.09^{\mathrm{de}}$ & $27.63 \pm 0.25^{\mathrm{dc}}$ & $20.78 \pm 0.17^{\mathrm{dc}}$ \\
\hline & $T_{10}$ & $4.09 \pm 0.13^{\mathrm{d}}$ & $15.82 \pm 0.19^{d}$ & $23.98 \pm 0.41^{\mathrm{c}}$ & $19.58 \pm 0.56^{\circ}$ & $7.18 \pm 0.53^{\mathrm{c}}$ & $13.35 \pm 0.51^{\mathrm{c}}$ & $25.68 \pm 0.51^{\mathrm{c}}$ & $19.51 \pm 0.50^{\mathrm{c}}$ \\
\hline
\end{tabular}


Table 5. Effects of E. heterophylla weeds density and distance on the shoot dry weight of A. esculentus (Var.'Kirikoi' and 'NHAe-40days')

\begin{tabular}{|c|c|c|c|c|c|c|c|c|c|}
\hline & \multirow{2}{*}{ Treatments } & \multicolumn{4}{|c|}{ Var. 'Kirikoi' } & \multicolumn{4}{|c|}{ Var. 'NHAe-40days' } \\
\hline & & $2 \mathrm{WAP}$ & 4 WAP & 6 WAP & $8 \mathrm{WAP}$ & $2 \mathrm{WAP}$ & $4 \mathrm{WAP}$ & 6 WAP & $8 \mathrm{WAP}$ \\
\hline \multirow{11}{*}{$\begin{array}{l}20 \mathrm{~cm} \text { weed } \\
\text { distance }\end{array}$} & $\mathrm{T}_{0}$ & $0.28 \pm 0.04^{a}$ & $1.37 \pm 0.01^{2}$ & $4.23 \pm 0.17^{\mathrm{a}}$ & $6.69 \pm 0.17^{a}$ & $0.23 \pm 0.02^{2}$ & $0.73 \pm 0.02^{2}$ & $2.18 \pm 0.05^{\mathrm{a}}$ & $2.91 \pm 0.07^{\mathrm{a}}$ \\
\hline & $T_{1}$ & $0.19 \pm 0.02^{\mathrm{b}}$ & $1.40 \pm 0.25^{\mathrm{a}}$ & $4.20 \pm 0.37^{a}$ & $6.59 \pm 0.49^{a}$ & $0.21 \pm 0.02^{\mathrm{a}}$ & $0.65 \pm 0.04^{\mathrm{ab}}$ & $1.96 \pm 0.12^{\mathrm{ab}}$ & $2.61 \pm 0.16^{\mathrm{ab}}$ \\
\hline & $\mathrm{T}_{2}$ & $0.20 \pm 0.01 \mathrm{c}$ & $1.14 \pm 0.06^{\mathrm{ab}}$ & $3.76 \pm 0.14^{\mathrm{ab}}$ & $5.90 \pm 0.24^{b}$ & $0.17 \pm 0.01^{\mathrm{b}}$ & $0.61 \pm 0.01^{\mathrm{b}}$ & $1.84 \pm 0.04^{\mathrm{b}}$ & $2.45 \pm 0.06^{b}$ \\
\hline & $\mathrm{T}_{3}$ & $0.12 \pm 0.01^{\mathrm{cd}}$ & $0.99 \pm 0.06^{\mathrm{bc}}$ & $3.35 \pm 0.08^{b}$ & $5.31 \pm 0.17^{b}$ & $0.15 \pm 0.00^{b c}$ & $0.50 \pm 0.04^{\mathrm{cd}}$ & $1.51 \pm 0.13^{\mathrm{cd}}$ & $2.01 \pm 0.17^{\mathrm{cd}}$ \\
\hline & $\mathrm{T}_{4}$ & $0.09 \pm 0.01 \mathrm{~cd}$ & $0.92 \pm 0.10^{\text {bde }}$ & $2.77 \pm 0.10^{c}$ & $4.47 \pm 0.09^{c}$ & $0.13 \pm 0.01^{\mathrm{bcd}}$ & $0.52 \pm 0.04^{c}$ & $1.56 \pm 0.11^{\mathrm{c}}$ & $2.08 \pm 0.14^{c}$ \\
\hline & $\mathrm{T}_{5}$ & $0.12 \pm 0.05^{c}$ & $0.96 \pm 0.07^{\mathrm{bcd}}$ & $2.74 \pm 0.15^{c}$ & $4.35 \pm 0.26^{c}$ & $0.12 \pm 0.02^{\mathrm{cd} k}$ & $0.49 \pm 0.04^{\mathrm{cdc}}$ & $1.46 \pm 0.11^{1 \mathrm{de}}$ & $1.95 \pm 0.15^{\mathrm{dcc}}$ \\
\hline & $\mathrm{T}_{6}$ & $0.06 \pm 0.01^{\mathrm{de}}$ & $0.82 \pm 0.03^{\mathrm{cde}}$ & $2.61 \pm 0.14^{c}$ & $4.04 \pm 0.26^{\mathrm{cd}}$ & $0.11 \pm 0.01^{\mathrm{cdc}}$ & $0.42 \pm 0.01^{\text {def }}$ & $1.26 \pm 0.03^{\mathrm{def}}$ & $1.68 \pm 0.04^{\mathrm{def}}$ \\
\hline & $\mathrm{T}_{7}$ & $0.05 \pm 0.01^{\mathrm{de}}$ & $0.70 \pm 0.03^{\mathrm{cdc}}$ & $2.34 \pm 0.06^{\mathrm{cd}}$ & $3.83 \pm 0.08^{\mathrm{cd}}$ & $0.10 \pm 0.01^{\mathrm{de}}$ & $0.47 \pm 0.03^{\text {cde }}$ & $1.40 \pm 0.10^{\mathrm{cdc}}$ & $1.87 \pm 0.13^{\mathrm{dec}}$ \\
\hline & $\mathrm{T}_{8}$ & $0.03 \pm 0.01^{\mathrm{de}}$ & $0.74 \pm 0.08^{c \mathrm{de}}$ & $2.35 \pm 0.18^{\mathrm{cd}}$ & $3.97 \pm 0.16^{\mathrm{cd}}$ & $0.09 \pm 0.01^{\text {ef }}$ & $0.43 \pm 0.02^{\text {def }}$ & $1.28 \pm 0.07^{\mathrm{def}}$ & $1.71 \pm 0.09^{\text {def }}$ \\
\hline & $\mathrm{T}_{9}$ & $0.03 \pm 0.01^{\mathrm{de}}$ & $0.65 \pm 0.05^{\mathrm{dc}}$ & $2.22 \pm 0.14^{\mathrm{cd}}$ & $3.75 \pm 0.19^{\text {cd }}$ & $0.05 \pm 0.01^{f}$ & $0.41 \pm 0.01^{\text {ef }}$ & $1.22 \pm 0.04^{\mathrm{cf}}$ & $1.63 \pm 0.06^{\text {cf }}$ \\
\hline & $\mathrm{T}_{10}$ & $0.02 \pm 0.00^{c}$ & $0.61 \pm 0.04^{e}$ & $2.00 \pm 0.10^{d}$ & $3.41 \pm 0.06^{\mathrm{d}}$ & $0.05 \pm 0.01^{f}$ & $0.36 \pm 0.02^{f}$ & $1.08 \pm 0.07^{\mathrm{f}}$ & $1.44 \pm 0.09^{f}$ \\
\hline \multirow{11}{*}{$\begin{array}{c}30 \mathrm{~cm} \text { weed } \\
\text { distance }\end{array}$} & $\mathrm{T}_{0}$ & $0.25 \pm 002^{a}$ & $1.34 \pm 0.03^{a}$ & $4.20 \pm 0.18^{\mathrm{a}}$ & $6.66 \pm 0.17^{\mathrm{a}}$ & $0.26 \pm 0.01^{2}$ & $0.76 \pm 0.05^{a}$ & $2.27 \pm 0.14^{a}$ & $3.03 \pm 0.19^{2}$ \\
\hline & $\mathrm{T}_{1}$ & $0.09 \pm 0.04^{\mathrm{b}}$ & $1.30 \pm 0.25^{\mathrm{a}}$ & $4.10 \pm 0.37^{\mathrm{bc}}$ & $6.49 \pm 0.48^{a}$ & $0.26 \pm 0.01^{2}$ & $0.70 \pm 0.06^{\mathrm{ab}}$ & $2.09 \pm 0.19^{\mathrm{ab}}$ & $2.79 \pm 0.25^{\mathrm{ab}}$ \\
\hline & $\mathrm{T}_{2}$ & $0.07 \pm 0.02^{\mathrm{b}}$ & $1.02 \pm 0.06^{\mathrm{b}}$ & $3.63 \pm 0.11^{c}$ & $5.77 \pm 0.22^{\mathrm{b}}$ & $0.24 \pm 0.01^{\mathrm{ab}}$ & $0.68 \pm 0.03^{\mathrm{ab}}$ & $2.05 \pm 0.10^{\mathrm{ab}}$ & $2.73 \pm 0.13^{\text {ab }}$ \\
\hline & $\mathrm{T}_{3}$ & $0.11 \pm 0.02^{\mathrm{b}}$ & $0.98 \pm 0.07^{\mathrm{bc}}$ & $3.34 \pm 0.09^{\mathrm{bc}}$ & $5.30 \pm 0.19^{b}$ & $0.23 \pm 0.01^{\text {bb }}$ & $0.59 \pm 0.04^{\mathrm{bc}}$ & $1.76 \pm 0.12^{\mathrm{bc}}$ & $2.35 \pm 0.16^{b c}$ \\
\hline & $\mathrm{T}_{4}$ & $0.11 \pm 0.02^{\mathrm{b}}$ & $0.94 \pm 0.08^{\mathrm{bc}}$ & $2.79 \pm 0.08^{c}$ & $4.49 \pm 0.10^{c}$ & $0.21 \pm 0.01^{\mathrm{bc}}$ & $0.60 \pm 0.03^{\mathrm{bc}}$ & $1.80 \pm 0.10^{\mathrm{bc}}$ & $2.40 \pm 0.14^{b c}$ \\
\hline & $\mathrm{T}_{5}$ & $0.13 \pm 0.03^{\mathrm{b}}$ & $0.97 \pm 0.03^{\mathrm{bc}}$ & $2.75 \pm 0.18^{c}$ & $4.36 \pm 0.27^{\mathrm{c}}$ & $0.19 \pm 0.01^{\mathrm{c}}$ & $0.56 \pm 0.06^{\mathrm{cd}}$ & $1.67 \pm 0.19^{\mathrm{dd}}$ & $2.23 \pm 0.25^{\mathrm{cd}}$ \\
\hline & $\mathrm{T}_{6}$ & $0.12 \pm 0.04^{\mathrm{b}}$ & $0.88 \pm 0.03^{\mathrm{bc}}$ & $2.67 \pm 0.10^{c}$ & $4.10 \pm 0.21^{\mathrm{cd}}$ & $0.15 \pm 0.01^{d}$ & $0.45 \pm 0.02^{\mathrm{dc}}$ & $1.36 \pm 0.07^{\mathrm{de}}$ & $1.81 \pm 0.09^{\mathrm{de}}$ \\
\hline & $\mathrm{T}_{7}$ & $0.08 \pm 0.01^{\mathrm{b}}$ & $0.73 \pm 0.02^{\mathrm{bc}}$ & $2.37 \pm 0.08^{\mathrm{dc}}$ & $3.85 \pm 0.09^{\mathrm{cd}}$ & $0.14 \pm 0.01^{d}$ & $0.50 \pm 0.03^{\text {de }}$ & $1.51 \pm 0.10^{\text {de }}$ & $2.01 \pm 0.14^{\mathrm{dde}}$ \\
\hline & $\mathrm{T}_{8}$ & $0.11 \pm 0.03^{\mathrm{b}}$ & $0.81 \pm 0.06^{\mathrm{bc}}$ & $2.43 \pm 0.15^{\mathrm{dc}}$ & $4.04 \pm 0.15^{\mathrm{cd}}$ & $0.14 \pm 0.01^{\mathrm{d}}$ & $0.48 \pm 0.03^{\mathrm{cdc}}$ & $1.43 \pm 0.08^{\mathrm{cdc}}$ & $1.91 \pm 0.11^{\mathrm{cdc}}$ \\
\hline & $\mathrm{T}_{9}$ & $0.13 \pm 0.04^{\mathrm{b}}$ & $0.75 \pm 0.02^{\mathrm{bc}}$ & $2.31 \pm 0.12^{\mathrm{dc}}$ & $3.84 \pm 0.16^{\mathrm{cd}}$ & $0.09 \pm 0.01^{\mathrm{e}}$ & $0.45 \pm 0.01^{\mathrm{dc}}$ & $1.34 \pm 0.03^{\mathrm{de}}$ & $1.79 \pm 0.04^{\mathrm{dc}}$ \\
\hline & $\mathrm{T}_{10}$ & $0.12 \pm 0.05^{\mathrm{b}}$ & $0.71 \pm 0.01^{\mathrm{c}}$ & $2.10 \pm 0.05^{\mathrm{c}}$ & $3.51 \pm 0.03^{\mathrm{d}}$ & $0.08 \pm 0.02^{\mathrm{c}}$ & $0.39 \pm 0.02^{\mathrm{c}}$ & $1.18 \pm 0.05^{c}$ & $1.57 \pm 0.07^{\mathrm{c}}$ \\
\hline \multirow{11}{*}{$\begin{array}{l}\text { Weed } \\
\text { density }\end{array}$} & $\mathrm{T}_{0}$ & $0.05 \pm 0.02^{a}$ & $1.14 \pm 0.07^{\mathrm{ab}}$ & $4.00 \pm 0.21^{a}$ & $6.46 \pm 0.19^{a}$ & $0.23 \pm 0.02^{ \pm}$ & $0.73 \pm 0.05^{a}$ & $2.19 \pm 0.15^{a}$ & $2.92 \pm 0.20^{2}$ \\
\hline & $\mathrm{T}_{1}$ & $0.05 \pm 0.01^{a}$ & $1.26 \pm 0.26^{a}$ & $4.06 \pm 0.38^{\mathrm{a}}$ & $6.45 \pm 0.49^{a}$ & $0.22 \pm 0.01^{a b}$ & $0.66 \pm 0.05^{a}$ & $1.97 \pm 0.14^{a}$ & $2.63 \pm 0.19^{a}$ \\
\hline & $\mathrm{T}_{2}$ & $0.09 \pm 0.03^{\mathrm{a}}$ & $1.03 \pm 0.06^{\mathrm{abc}}$ & $3.65 \pm 0.10^{\mathrm{ab}}$ & $5.79 \pm 0.20^{\mathrm{ab}}$ & $0.19 \pm 0.01^{\mathrm{bc}}$ & $0.63 \pm 0.02^{\mathrm{ab}}$ & $1.89 \pm 0.05^{\mathrm{ab}}$ & $2.52 \pm 0.07^{\mathrm{ab}}$ \\
\hline & $\mathrm{T}_{3}$ & $0.06 \pm 0.01^{a}$ & $0.93 \pm 0.06^{\text {bcd }}$ & $3.30 \pm 0.07^{\mathrm{b}}$ & $5.25 \pm 0.16^{c}$ & $0.16 \pm 0.01^{\mathrm{cd}}$ & $0.51 \pm 0.05^{\mathrm{cd}}$ & $1.54 \pm 0.14^{\mathrm{cd}}$ & $2.05 \pm 0.19^{\mathrm{cd}}$ \\
\hline & $\mathrm{T}_{4}$ & $0.07 \pm 0.00^{2}$ & $0.90 \pm 0.09^{\mathrm{bcd}}$ & $2.74 \pm \pm 0.09^{c}$ & $4.45 \pm 0.09^{\circ}$ & $0.15 \pm 0.01^{\text {de }}$ & $0.53 \pm 0.04^{c}$ & $1.60 \pm 0.13^{\mathrm{bc}}$ & $2.13 \pm 0.18^{b c}$ \\
\hline & $\mathrm{T}_{5}$ & $0.08 \pm 0.02^{a}$ & $0.93 \pm 0.03^{\text {bcd }}$ & $2.71 \pm 0.16^{c}$ & $4.32 \pm 0.27^{c}$ & $0.13 \pm 0.02^{\text {def }}$ & $0.49 \pm 0.04^{\mathrm{cd}}$ & $1.48 \pm 0.13^{\mathrm{cd}}$ & $1.97 \pm 0.18^{\text {cd }}$ \\
\hline & $\mathrm{T}_{6}$ & $0.08 \pm 0.03^{a}$ & $0.85 \pm 0.05^{\text {bcd }}$ & $2.63 \pm 0.14^{c}$ & $4.07 \pm 0.26^{\mathrm{cd}}$ & $0.10 \pm 0.01^{\sqrt{5}}$ & $0.41 \pm 0.01^{\mathrm{dc}}$ & $1.22 \pm 0.13^{\mathrm{de}}$ & $1.63 \pm 0.06^{\mathrm{de}}$ \\
\hline & $\mathrm{T}_{7}$ & $0.09 \pm 0.03^{\mathrm{a}}$ & $0.74 \pm 0.06^{\mathrm{cd}}$ & $2.38 \pm 0.08^{\text {cd }}$ & $3.86 \pm 0.11^{\mathrm{cd}}$ & $0.11 \pm 0.02^{\mathrm{c}}$ & $0.48 \pm 0.06^{\mathrm{cdc}}$ & $1.43 \pm 0.17^{\mathrm{ddc}}$ & $1.91 \pm 0.23^{\mathrm{cdc}}$ \\
\hline & $\mathrm{T}_{8}$ & $0.05 \pm 0.00^{a}$ & $0.75 \pm 0.08^{c d}$ & $2.37 \pm 0.17^{\mathrm{cd}}$ & $3.98 \pm 0.16^{\mathrm{cd}}$ & $0.08 \pm 0.01^{\text {gh }}$ & $0.42 \pm 0.02^{\mathrm{cdc}}$ & $1.25 \pm 0.07^{\mathrm{ddc}}$ & $1.67 \pm 0.09^{\mathrm{ddc}}$ \\
\hline & $\mathrm{T}_{9}$ & $0.08 \pm 0.02^{\mathrm{a}}$ & $0.70 \pm 0.06^{d}$ & $2.26 \pm 0.13^{\mathrm{cd}}$ & $3.79 \pm 0.20^{\text {dd }}$ & $0.05 \pm 0.00^{\mathrm{h}}$ & $0.41 \pm 0.01^{\mathrm{de}}$ & $1.22 \pm 0.03^{\mathrm{dc}}$ & $1.63 \pm 0.04^{\mathrm{de}}$ \\
\hline & $\mathrm{T}_{10}$ & $0.05 \pm 0.00^{2}$ & $0.64 \pm 0.04^{\mathrm{d}}$ & $2.03 \pm 0.10^{d}$ & $3.44 \pm 0.06^{d}$ & $0.05 \pm 0.00^{\mathrm{h}}$ & $0.36 \pm 0.03^{\mathrm{c}}$ & $1.09 \pm 0.08^{\mathrm{c}}$ & $1.45 \pm 0.10^{c}$ \\
\hline
\end{tabular}

Values represent Means \pm SEM, $\mathrm{n}=3$, values with same superscript along the column are significantly the same at $\mathrm{p}<0.05 ; \mathrm{T}_{0}=\mathrm{control}_{1} \mathrm{~T}_{1}=1$ weed, $\mathrm{T}_{1}=1$ weed plant ${ }^{-1}$, $\mathrm{T}_{2}=2$ weeds plant ${ }^{-1}, \mathrm{~T}_{3}=3$ weeds plant ${ }^{-1}, \mathrm{~T}_{4}=4$ weeds plant ${ }^{-1}, \mathrm{~T}_{5}=5$ weeds plant ${ }^{-1}, \mathrm{~T}_{6}=6$ weeds plant ${ }^{-1}, \mathrm{~T}_{7}=7$ weed plant $^{-1}, \mathrm{~T}_{8}=8 \mathrm{weed} \mathrm{plant}^{-1}, \mathrm{~T}_{9}=9$ weed $\mathrm{plant}^{-1}, \mathrm{~T}_{10}=$ 10 weed plant ${ }^{-1}$

Table 6. Effects of E. heterophylla weeds density and distance on the yield of $A$. esculentus (Var.'Kirikoi')

\begin{tabular}{|c|c|c|c|c|c|c|c|}
\hline & Treatment & Average Fruit Number & Average Groove Number & $\begin{array}{l}\text { Average Pod Length } \\
(\mathrm{cm})\end{array}$ & Average Pod Girth $(\mathrm{cm})$ & $\begin{array}{l}\text { Average Fresh Pod } \\
\text { Weight Weight (g) }\end{array}$ & $\begin{array}{l}\text { Average Dry Pod Weight } \\
\text { (g) }\end{array}$ \\
\hline \multirow{11}{*}{$\begin{array}{l}20 \mathrm{~cm} \text { weed } \\
\text { distance }\end{array}$} & $\mathrm{T}_{0}$ & $5.67 \pm 0.33^{2}$ & $45.00 \pm 3.06^{a}$ & $39.03 \pm 4.07^{\mathrm{a}}$ & $22.17 \pm 1.34^{a}$ & $37.11 \pm 1.03^{a}$ & $15.90 \pm 0.44 a$ \\
\hline & $\mathrm{T}_{1}$ & $5.00 \pm 0.58^{\mathrm{bb}}$ & $36.33 \pm 1.20^{b}$ & $28.20 \pm 1.44^{b}$ & $19.67 \pm 1.16^{\mathrm{ab}}$ & $31.99 \pm 2.26^{6}$ & $13.71 \pm 0.97^{b}$ \\
\hline & $\mathrm{T}_{2}$ & $4.67 \pm 0.67^{\mathrm{abc}}$ & $34.00 \pm 2.31^{\mathrm{b}}$ & $26.93 \pm 1.68^{\mathrm{bc}}$ & $18.70 \pm 0.71^{\mathrm{b}}$ & $32.64 \pm 1.73^{b}$ & $13.99 \pm 0.74^{\mathrm{b}}$ \\
\hline & $\mathrm{T}_{3}$ & $4.67 \pm 0.33^{\mathrm{bbc}}$ & $30.67 \pm 0.67^{\mathrm{bc}}$ & $21.97 \pm 1.60^{\mathrm{cd}}$ & $16.80 \pm 1.07^{\mathrm{b}}$ & $27.41 \pm 2.03^{c}$ & $11.75 \pm 0.87^{\circ}$ \\
\hline & $\mathrm{T}_{4}$ & $4.33 \pm 0.33^{\text {bdd }}$ & $35.67 \pm 1.86^{b}$ & $19.37 \pm 2.58^{\text {de }}$ & $14.00 \pm 1.16^{c}$ & $24.88 \pm 0.73^{\mathrm{cd}}$ & $10.66 \pm 0.31^{\mathrm{cd}}$ \\
\hline & $\mathrm{T}_{5}$ & $4.00 \pm 0.00^{\text {bed }}$ & $26.67 \pm 1.67^{\mathrm{ddc}}$ & $17.03 \pm 1.81^{\mathrm{def}}$ & $12.37 \pm 0.88^{\text {cde }}$ & $24.62 \pm 1.41^{\mathrm{cd}}$ & $10.55 \pm 0.60^{\mathrm{cd}}$ \\
\hline & $\mathrm{T}_{6}$ & $3.67 \pm 0.33^{\text {dd }}$ & $27.67 \pm 1.86^{\text {cd }}$ & $19.70 \pm 1.34^{\mathrm{de}}$ & $13.87 \pm 1.03^{\mathrm{cd}}$ & $24.84 \pm 0.67^{\mathrm{cd}}$ & $10.65 \pm 0.29^{\mathrm{dd}}$ \\
\hline & $\mathrm{T}_{7}$ & $3.33 \pm 0.33^{\mathrm{d}}$ & $21.67 \pm 1.20^{\text {def }}$ & $15.23 \pm 1.21^{\text {ef }}$ & $12.53 \pm 0.70^{\text {cde }}$ & $22.00 \pm 1.83^{\mathrm{de}}$ & $9.43 \pm 0.78^{\mathrm{de}}$ \\
\hline & $\mathrm{T}_{8}$ & $3.67 \pm 0.33^{\mathrm{cd}}$ & $21.00 \pm 2.65^{\text {cf }}$ & $13.87 \pm 1.77^{\text {ef }}$ & $10.93 \pm 0.57^{\mathrm{dcf} f}$ & $22.41 \pm 1.28^{\mathrm{dc}}$ & $9.61 \pm 0.55^{\mathrm{dc}}$ \\
\hline & $\mathrm{T}_{9}$ & $3.33 \pm 0.33^{\mathrm{d}}$ & $19.33 \pm 1.86^{\mathrm{F}}$ & $13.23 \pm 0.98^{\text {cf }}$ & $10.43 \pm 0.79^{\mathrm{cf}}$ & $20.68 \pm 1.09^{\mathrm{de}}$ & $8.86 \pm 0.47^{\mathrm{dc}}$ \\
\hline & $\mathrm{T}_{10}$ & $3.33 \pm 0.33^{\mathrm{d}}$ & $16.00 \pm 2.00^{f}$ & $11.47 \pm 1.13^{f}$ & $8.63 \pm 0.59^{f}$ & $19.50 \pm 1.37^{\mathrm{c}}$ & $8.36 \pm 0.59^{\circ}$ \\
\hline \multirow{11}{*}{$\begin{array}{l}30 \mathrm{~cm} \text { weed } \\
\text { distance }\end{array}$} & $\mathrm{T}_{0}$ & $7.00 \pm 0.58^{a}$ & $45.00 \pm 2.00^{2}$ & $38.77 \pm 4.23^{a}$ & $25.53 \pm 1.73^{a}$ & $37.30 \pm 1.07^{2}$ & $15.99 \pm 0.46^{a}$ \\
\hline & $\mathrm{T}_{1}$ & $6.67 \pm 0.33^{3 \mathrm{~b}}$ & $41.00 \pm 3.00^{\mathrm{ab}}$ & $23.10 \pm 1.93^{\mathrm{bcd}}$ & $17.17 \pm 2.22^{\mathrm{abc}}$ & $36.35 \pm 0.43^{\mathrm{ab}}$ & $15.58 \pm 0.18^{\mathrm{ab}}$ \\
\hline & $\mathrm{T}_{2}$ & $5.67 \pm 0.33^{\mathrm{bc}}$ & $38.67 \pm 3.18^{b x}$ & $32.70 \pm 4.84^{\mathrm{ab}}$ & $26.47 \pm 4.40^{\mathrm{a}}$ & $32.84 \pm 1.06^{c}$ & $14.08 \pm 0.46^{c}$ \\
\hline & $\mathrm{T}_{3}$ & $5.67 \pm 0.33^{\mathrm{bc}}$ & $34.00 \pm 2.52^{\mathrm{cdc}}$ & $25.73 \pm 1.77^{b c}$ & $20.57 \pm 1.19^{\mathrm{ab}}$ & $33.45 \pm 0.80^{\mathrm{bc}}$ & $14.34 \pm 0.34^{c}$ \\
\hline & $\mathrm{T}_{4}$ & $5.67 \pm 0.33^{\text {bc }}$ & $36.00 \pm 2.00^{\mathrm{bcd}}$ & $22.43 \pm 3.43^{\mathrm{cd}}$ & $17.07 \pm 4.25^{\mathrm{abc}}$ & $31.94 \pm 0.64^{c}$ & $13.69 \pm 0.27^{\mathrm{c}}$ \\
\hline & $\mathrm{T}_{5}$ & $5.33 \pm 0.33^{\text {cd }}$ & $30.67 \pm 1.33^{\mathrm{dc}}$ & $15.33 \pm 3.54^{\mathrm{de}}$ & $10.67 \pm 5.16^{\mathrm{bcd}}$ & $31.87 \pm 0.60^{\circ}$ & $13.66 \pm 0.26^{c}$ \\
\hline & $\mathrm{T}_{6}$ & $5.00 \pm 0.00^{\mathrm{cdc}}$ & $28.33 \pm 1.45^{c f}$ & $9.77 \pm 3.27^{\mathrm{c}}$ & $3.93 \pm 2.96^{\mathrm{d}}$ & $27.32 \pm 1.56^{\mathrm{d}}$ & $11.71 \pm 0.67^{\mathrm{d}}$ \\
\hline & $\mathrm{T}_{7}$ & $5.00 \pm 0.00^{\mathrm{cdc}}$ & $24.00 \pm 1.00^{f_{8}^{8}}$ & $16.90 \pm 2.19^{\mathrm{dec}}$ & $14.20 \pm 1.29^{\mathrm{bc}}$ & $26.75 \pm 1.54^{d}$ & $11.46 \pm 0.66^{d}$ \\
\hline & $\mathrm{T}_{8}$ & $4.67 \pm 0.33^{\mathrm{cdc}}$ & $23.00 \pm 1.53^{f_{g}}$ & $21.70 \pm 0.91^{\mathrm{cd}}$ & $18.77 \pm 0.48^{\mathrm{abc}}$ & $24.80 \pm 1.65^{\mathrm{dc}}$ & $10.63 \pm 0.71^{\text {de }}$ \\
\hline & $\mathrm{T}_{9}$ & $4.33 \pm 0.33^{\mathrm{de}}$ & $24.33 \pm 0.83^{\frac{58}{8}}$ & $11.23 \pm 3.85^{c}$ & $8.43 \pm 4.05^{\mathrm{cd}}$ & $22.31 \pm 1.13^{\text {ef }}$ & $9.56 \pm 0.48^{e f}$ \\
\hline & $\mathrm{T}_{10}$ & $4.00 \pm 0.00^{c}$ & $20.00 \pm 0.00^{\mathrm{g}}$ & $14.40 \pm 2.17^{\mathrm{de}}$ & $11.57 \pm 2.52^{\mathrm{bcd}}$ & $20.72 \pm 1.03^{\mathrm{f}}$ & $8.88 \pm 0.44^{f}$ \\
\hline \multirow{11}{*}{$\begin{array}{l}\text { Weed } \\
\text { density }\end{array}$} & $\mathrm{T}_{0}$ & $6.33 \pm 0.33^{2}$ & $42.00 \pm 1.53^{a}$ & $36.03 \pm 2.70^{\mathrm{a}}$ & $19.17 \pm 2.76 \mathrm{a}$ & $36.66 \pm 0.91^{2}$ & $15.71 \pm 0.39^{\mathrm{a}}$ \\
\hline & $T_{1}$ & $4.67 \pm 0.33^{\mathrm{b}}$ & $35.00 \pm 1.53^{b}$ & $26.87 \pm 4.01^{\mathrm{b}}$ & $18.33 \pm 3.76^{2}$ & $35.00 \pm 1.44^{2}$ & $15.00 \pm 0.62^{\mathrm{a}}$ \\
\hline & $\mathrm{T}_{2}$ & $4.33 \pm 0.33^{\mathrm{bc}}$ & $33.33 \pm 1.45^{\mathrm{bc}}$ & $26.27 \pm 4.13^{b}$ & $18.03 \pm 4.09^{2}$ & $30.44 \pm 1.32^{\mathrm{b}}$ & $13.05 \pm 0.57^{b}$ \\
\hline & $\mathrm{T}_{3}$ & $4.00 \pm 0.00^{\mathrm{bcd}}$ & $30.67 \pm 1.76^{\mathrm{bcd}}$ & $21.97 \pm 0.92^{\mathrm{bc}}$ & $16.80 \pm 1.35^{\mathrm{ab}}$ & $30.83 \pm 1.04^{\mathrm{b}}$ & $13.21 \pm 0.45^{b}$ \\
\hline & $\mathrm{T}_{4}$ & $3.67 \pm 0.33^{\text {cde }}$ & $29.33 \pm 1.67^{\mathrm{dde}}$ & $13.03 \pm 2.29^{d}$ & $7.67 \pm 3.37^{c}$ & $27.91 \pm 1.00^{\mathrm{b}}$ & $11.96 \pm 0.43^{b}$ \\
\hline & $\mathrm{T}_{5}$ & $3.67 \pm 0.33^{\text {cde }}$ & $27.67 \pm 0.88^{\mathrm{dc}}$ & $18.03 \pm 1.75^{\mathrm{cd}}$ & $13.37 \pm 0.76^{\text {thc }}$ & $24.57 \pm 0.71^{c}$ & $10.53 \pm 0.30^{c}$ \\
\hline & $\mathrm{T}_{6}$ & $3.33 \pm 0.33^{\mathrm{de}}$ & $25.00 \pm 0.58^{\text {cf }}$ & $17.03 \pm 0.12^{\mathrm{cd}}$ & $11.20 \pm 0.44^{\mathrm{bc}}$ & $24.26 \pm 1.26^{c}$ & $10.40 \pm 0.54^{\mathrm{c}}$ \\
\hline & $\mathrm{T}_{7}$ & $3.00 \pm 0.00^{\mathrm{c}}$ & $23.00 \pm 1.53^{f}$ & $16.57 \pm 2.99^{\mathrm{cd}}$ & $13.97 \pm 2.35^{\mathrm{abc}}$ & $22.33 \pm 1.17^{c}$ & $9.57 \pm 0.50^{c}$ \\
\hline & $\mathrm{T}_{8}$ & $2.33 \pm 0.33^{\text {fg }}$ & $18.00 \pm 1.15^{8}$ & $10.87 \pm 2.14^{d}$ & $7.93 \pm 1.67^{\mathrm{c}}$ & $19.11 \pm 0.64^{d}$ & $8.19 \pm 0.27^{d}$ \\
\hline & $\mathrm{T}_{9}$ & $2.33 \pm 0.33^{\text {fg }}$ & $17.67 \pm 1.76^{3}$ & $11.57 \pm 1.99^{d}$ & $8.77 \pm 2.18^{\mathrm{bc}}$ & $18.79 \pm 0.85^{d}$ & $8.05 \pm 0.36^{d}$ \\
\hline & $T_{10}$ & $2.00 \pm 0.00^{\mathrm{s}}$ & $14.00 \pm 1.53^{3}$ & $11.13 \pm 1.15^{d}$ & $9.53 \pm 0.52^{b c}$ & $17.79 \pm 0.88^{d}$ & $7.62 \pm 0.38^{d}$ \\
\hline
\end{tabular}

Values represent Means \pm SEM, $\mathrm{n}=3$, values with same superscript along the column are significantly the same at $\mathrm{p}<0.05 ; \mathrm{T}_{0}=\mathrm{control}_{1} \mathrm{~T}_{1}=1$ weed, $\mathrm{T}_{1}=1$ weed plant ${ }^{-1}$,

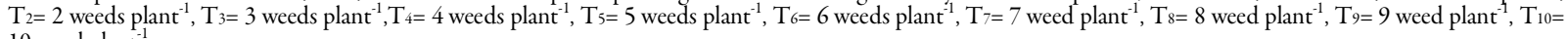
10 weed plant ${ }^{-1}$ 
Table 7. Effects of E. heterophylla weeds density and distance on the yield of A. esculentus (Var. 'NHA-e 40days')

\begin{tabular}{|c|c|c|c|c|c|c|c|}
\hline & Treatment & Fruit Number & Average Groove Number & $\begin{array}{l}\text { Average Pod Length } \\
(\mathrm{cm})\end{array}$ & Average Pod Girth $(\mathrm{cm})$ & $\begin{array}{l}\text { Average Fresh Pod } \\
\text { Weight }(\mathrm{g})\end{array}$ & $\begin{array}{c}\text { Average } \\
\text { Dry Pod Weight (g) }\end{array}$ \\
\hline \multirow{11}{*}{$\begin{array}{l}20 \mathrm{~cm} \text { weed } \\
\text { distance }\end{array}$} & $T_{0}$ & $5.33 \pm 0.33^{2}$ & $39.67 \pm 1.76^{2}$ & $35.26 \pm 1.57^{\alpha}$ & $27.42 \pm 1.22^{2}$ & $28.51 \pm 1.58^{\mathrm{a}}$ & $12.22 \pm 0.68^{\mathrm{a}}$ \\
\hline & $\mathrm{T}_{1}$ & $5.00 \pm 0.00^{2}$ & $35.67 \pm 1.45^{\mathrm{ab}}$ & $31.70 \pm 1.29^{\mathrm{ab}}$ & $24.66 \pm 1.01^{\mathrm{ab}}$ & $28.18 \pm 1.15^{\mathrm{ab}}$ & $12.08 \pm 0.49^{9 \mathrm{~b}}$ \\
\hline & $\mathrm{T}_{2}$ & $4.67 \pm 0.33^{\mathrm{ab}}$ & $31.67 \pm 2.40^{\mathrm{bc}}$ & $28.15 \pm 2.14^{\mathrm{bc}}$ & $21.89 \pm 1.66^{\mathrm{bc}}$ & $25.02 \pm 1.90^{\mathrm{alc}}$ & $10.72 \pm 0.81^{\mathrm{abc}}$ \\
\hline & $\mathrm{T}_{3}$ & $4.67 \pm 0.33^{\mathrm{ab}}$ & $29.33 \pm 1.86^{\mathrm{bc}}$ & $26.07 \pm 1.65^{c}$ & $20.28 \pm 1.28^{\mathrm{bc}}$ & $23.17 \pm 1.47^{\mathrm{bc}}$ & $9.93 \pm 0.63^{\mathrm{bc}}$ \\
\hline & $\mathrm{T}_{4}$ & $4.67 \pm 0.33^{\text {ab }}$ & $26.67 \pm 2.19^{c}$ & $23.70 \pm 1.95^{c}$ & $18.43 \pm 1.51^{c}$ & $21.07 \pm 1.73^{c}$ & $9.03 \pm 0.74^{c}$ \\
\hline & $\mathrm{T}_{5}$ & $4.00 \pm 0.00^{\mathrm{bc}}$ & $27.00 \pm 1.53^{c}$ & $24.00 \pm 1.36^{c}$ & $18.67 \pm 1.06^{c}$ & $21.33 \pm 1.21^{\mathrm{c}}$ & $9.14 \pm 0.52^{c}$ \\
\hline & $\mathrm{T}_{6}$ & $3.67 \pm 0.33^{\mathrm{cd}}$ & $28.33 \pm 0.33^{c}$ & $25.19 \pm 0.29^{b c}$ & $19.59 \pm 0.23^{c}$ & $22.38 \pm 0.26^{c}$ & $9.59 \pm 0.11^{\mathrm{c}}$ \\
\hline & $\mathrm{T}_{7}$ & $3.33 \pm 0.33^{\text {cd }}$ & $31.33 \pm 3.76^{\mathrm{bc}}$ & $27.85 \pm 3.34^{\mathrm{bc}}$ & $21.66 \pm 2.60^{\mathrm{bc}}$ & $24.75 \pm 2.97^{\mathrm{abc}}$ & $10.61 \pm 1.27^{\mathrm{abc}}$ \\
\hline & $\mathrm{T}_{8}$ & $3.33 \pm 0.33^{\mathrm{cd}}$ & $18.67 \pm 1.76^{\mathrm{d}}$ & $16.59 \pm 1.57^{d}$ & $12.90 \pm 1.22^{\mathrm{d}}$ & $14.75 \pm 1.39^{d}$ & $6.32 \pm 0.60^{d}$ \\
\hline & $\mathrm{T}_{9}$ & $3.00 \pm 0.00^{\mathrm{d}}$ & $17.00 \pm 1.53^{\mathrm{d}}$ & $15.11 \pm 1.36^{\mathrm{d}}$ & $11.75 \pm 1.06^{\mathrm{d}}$ & $13.43 \pm 1.21^{\mathrm{d}}$ & $5.76 \pm 0.52^{d}$ \\
\hline & $\mathrm{T}_{10}$ & $3.00 \pm 0.00^{d}$ & $12.33 \pm 2.33 \mathrm{~d}$ & $10.96 \pm 2.07^{\mathrm{d}}$ & $8.52 \pm 1.61^{\mathrm{d}}$ & $9.74 \pm 1.84^{d}$ & $4.18 \pm 0.79^{d}$ \\
\hline \multirow{11}{*}{$\begin{array}{c}30 \mathrm{~cm} \text { weed } \\
\text { distance }\end{array}$} & $T_{0}$ & $5.67 \pm 0.33^{a}$ & $39.33 \pm 1.20^{\mathrm{a}}$ & $34.96 \pm 1.07^{\mathrm{a}}$ & $27.19 \pm 0.83^{2}$ & $28.38 \pm 2.15^{a}$ & $12.16 \pm 0.92^{2}$ \\
\hline & $\mathrm{T}_{1}$ & $5.33 \pm 0.33^{a}$ & $31.33 \pm 1.76^{c}$ & $27.85 \pm 1.57^{c}$ & $21.66 \pm 1.22^{c}$ & $24.75 \pm 1.39^{a}$ & $10.61 \pm 0.60^{b}$ \\
\hline & $\mathrm{T}_{2}$ & $5.33 \pm 0.33^{a}$ & $35.67 \pm 0.88^{b}$ & $31.70 \pm 0.78^{b}$ & $24.66 \pm 0.61^{b}$ & $28.18 \pm 0.70^{\mathrm{a}}$ & $12.08 \pm 0.30^{\mathrm{a}}$ \\
\hline & $\mathrm{T}_{3}$ & $5.33 \pm 0.33^{a}$ & $29.33 \pm 0.88^{\mathrm{cd}}$ & $26.08 \pm 0.78^{c d}$ & $20.28 \pm 0.61^{\mathrm{cd}}$ & $23.17 \pm 0.70^{\mathrm{bc}}$ & $9.93 \pm 0.30^{b x}$ \\
\hline & $\mathrm{T}_{4}$ & $5.00 \pm 0.00^{\mathrm{ab}}$ & $29.67 \pm 1.76^{\mathrm{cd}}$ & $26.37 \pm 1.57^{\mathrm{cd}}$ & $20.51 \pm 1.22^{\mathrm{cd}}$ & $23.44 \pm 1.39^{b}$ & $10.04 \pm 0.60^{\mathrm{b}}$ \\
\hline & $\mathrm{T}_{5}$ & $5.00 \pm 0.00^{\mathrm{ab}}$ & $28.33 \pm 1.45^{\mathrm{dc}}$ & $25.19 \pm 1.29^{\text {cde }}$ & $19.59 \pm 1.00^{\mathrm{dde}}$ & $22.38 \pm 1.15^{\mathrm{bcd}}$ & $9.59 \pm 0.49^{\mathrm{bcd}}$ \\
\hline & $\mathrm{T}_{6}$ & $4.33 \pm 0.33 \mathrm{bc}$ & $29.67 \pm 1.20^{\mathrm{cd}}$ & $26.37 \pm 1.07^{\mathrm{cd}}$ & $20.51 \pm 0.83^{\text {cd }}$ & $23.44 \pm 0.95^{\mathrm{b}}$ & $10.05 \pm 0.41^{\mathrm{b}}$ \\
\hline & $\mathrm{T}_{7}$ & $4.33 \pm 0.33 \mathrm{bc}$ & $28.67 \pm 0.88^{\mathrm{cd}}$ & $25.48 \pm 0.78^{\mathrm{cd}}$ & $19.82 \pm 0.61^{\mathrm{cd}}$ & $22.65 \pm 0.70^{\mathrm{kcd}}$ & $9.71 \pm 0.30^{\mathrm{bcd}}$ \\
\hline & $\mathrm{T}_{8}$ & $4.33 \pm 0.33^{\mathrm{bc}}$ & $26.67 \pm 0.67^{\text {def }}$ & $23.70 \pm 0.59^{\mathrm{def}}$ & $18.44 \pm 0.46^{\mathrm{dkf}}$ & $21.07 \pm 0.53^{\text {bcd }}$ & $9.03 \pm 0.23^{\text {bdd }}$ \\
\hline & $\mathrm{T}_{9}$ & $4.00 \pm 0.00^{c}$ & $24.67 \pm 1.33^{\text {ef }}$ & $21.93 \pm 1.18^{\text {ef }}$ & $17.06 \pm 0.92^{\text {ef }}$ & $19.49 \pm 1.05^{\mathrm{cd}}$ & $8.35 \pm 0.45^{\mathrm{cd}}$ \\
\hline & $\mathrm{T}_{10}$ & $3.67 \pm 0.33^{c}$ & $24.00 \pm 1.15^{\mathrm{f}}$ & $21.33 \pm 1.02^{f}$ & $16.59 \pm 0.80^{f}$ & $18.96 \pm 0.91^{d}$ & $8.13 \pm 0.39^{d}$ \\
\hline \multirow{11}{*}{$\begin{array}{l}\text { Weed } \\
\text { density }\end{array}$} & $\mathrm{T}_{0}$ & $5.33 \pm 0.33^{a}$ & $38.67 \pm 1.76^{a}$ & $34.37 \pm 1.57^{\lambda}$ & $26.73 \pm 1.22^{\mathrm{a}}$ & $28.05 \pm 3.09^{a}$ & $12.02 \pm 1.32^{4}$ \\
\hline & $\mathrm{T}_{1}$ & $4.67 \pm 0.33^{\text {ab }}$ & $33.33 \pm 0.33 b$ & $29.63 \pm 0.30^{\mathrm{b}}$ & $23.04 \pm 0.23^{b}$ & $26.33 \pm 0.26^{\mathrm{b}}$ & $11.28 \pm 0.11^{\mathrm{ab}}$ \\
\hline & $\mathrm{T}_{2}$ & $4.00 \pm 0.58^{\mathrm{bc}}$ & $31.00 \pm 1.53^{\mathrm{bc}}$ & $27.56 \pm 1.36^{\mathrm{bc}}$ & $21.43 \pm 1.06^{\mathrm{bc}}$ & $24.49 \pm 1.21^{\mathrm{alc}}$ & $10.50 \pm 0.52^{\mathrm{scc}}$ \\
\hline & $\mathrm{T}_{3}$ & $3.67 \pm 0.33^{\text {bcd }}$ & $30.00 \pm 1.15^{\mathrm{bc}}$ & $26.67 \pm 1.02^{b c}$ & $20.74 \pm 0.80^{\mathrm{bc}}$ & $23.70 \pm 0.91^{\mathrm{bc}}$ & $10.16 \pm 0.39^{\mathrm{bc}}$ \\
\hline & $\mathrm{T}_{4}$ & $3.33 \pm 0.33^{\mathrm{cdc}}$ & $29.00 \pm 0.58^{\mathrm{cd}}$ & $25.78 \pm 0.51^{\mathrm{cd}}$ & $20.05 \pm 0.40^{\mathrm{cd}}$ & $22.91 \pm 0.46^{\mathrm{bcd}}$ & $9.82 \pm 0.20^{\mathrm{bcd}}$ \\
\hline & $\mathrm{T}_{5}$ & $3.33 \pm 0.33^{\text {de }}$ & $26.33 \pm 0.88 \mathrm{de}$ & $23.41 \pm 0.78^{\mathrm{de}}$ & $18.21 \pm 0.61^{\mathrm{de}}$ & $20.80 \pm 0.70^{\text {de }}$ & $8.92 \pm 0.30^{\text {cde }}$ \\
\hline & $\mathrm{T}_{6}$ & $3.33 \pm 0.33^{\mathrm{dcc}}$ & $25.00 \pm 1.53^{\text {cf }}$ & $22.22 \pm 1.36^{\text {cf }}$ & $17.29 \pm 1.06^{\mathrm{cf}}$ & $19.75 \pm 1.21^{\text {def }}$ & $8.46 \pm 0.52^{\mathrm{def}}$ \\
\hline & $\mathrm{T}_{7}$ & $3.00 \pm 0.00^{\mathrm{cdc}}$ & $24.67 \pm 0.67^{\text {cf }}$ & $21.92 \pm 0.59^{\text {ff }}$ & $17.05 \pm 0.46^{\mathrm{ff}}$ & $19.49 \pm 0.53^{\text {def }}$ & $8.35 \pm 0.22^{\text {def }}$ \\
\hline & $\mathrm{T}_{8}$ & $2.67 \pm 0.33^{\mathrm{de}}$ & $21.00 \pm 0.58^{g}$ & $18.67 \pm 0.51^{\mathrm{g}}$ & $14.52 \pm 0.40^{8}$ & $16.59 \pm 0.46^{\mathrm{f}}$ & $7.11 \pm 0.20^{f}$ \\
\hline & $\mathrm{T}_{9}$ & $2.67 \pm 0.33^{\text {de }}$ & $22.67 \pm 0.88^{f_{s}}$ & $20.15 \pm 0.78^{\text {rg }}$ & $15.67 \pm 0.61^{f_{g}}$ & $17.91 \pm 0.70^{\text {ff }}$ & $7.68 \pm 0.30^{\text {ff }}$ \\
\hline & $\mathrm{T}_{10}$ & $2.33 \pm 0.33^{c}$ & $20.67 \pm 1.20 \mathrm{~g}$ & $18.37 \pm 1.07^{8}$ & $14.29 \pm 0.83^{z}$ & $16.33 \pm 0.95^{f}$ & $7.00 \pm 0.41^{f}$ \\
\hline
\end{tabular}

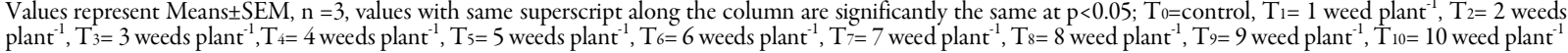

\section{References}

Amjad A, Anjum MA, Hussain S (2002). Effect of different sowing dates and various doses of fertilizers on juvenility and productivity of okra. Pakistan Journal of Agricultural Science 38(1-2):29-32.

Askew SD, Wilcut JW, Cranmer JR (2002). Cotton (Gossypium hirsutum. L.) and weed response to flumioxazin applied pre-plant and postemergence directed. Weed Technology 16(1):184-190.

Ayeni AO, Oyekan PO (1992). Weed control in soybean (Glycine maxL.) (Merr) in Nigeria. Tropical Oil SeedJournal 1:43-52.

Barrentine WL, Oliver LR (1997).Competitive threshold levels and control of common cocklebur in soybean. Mass Agricultural Experimental Station and Arkansans Agricultural Experimental Station Bull. McGuire.No83,pp 27.

Carvalho LB, Binaco S, Guzzo CD (2010). Interference of Euphorbia heterophylla in the growth and macro nutrient accumulation of soybean. PlantaDaninha28(1):33-39.

Chadha K.L (2002). Hand book of horticulture. Indian Council of Agricultural Research pp 427.

Falodun EJ, Ogedegbe SA (2006). Effects of planting spacing and harvest intervals on growth, yield and quality of okra (Abelmoschus esculentus $(\mathrm{L})$ Moench).). Pakistan Journal of Science and Industrial Research 39(5):225-308.
Ibe AO (2005). Effects of NPK fertilizers on quality of okra (Abelmoschus esculentus (L.) Moench) in an ultisol, Southern Nigeria; Unpublis he d Ph.D. Thesis, Department of Crop/Science, Rivers State University of Science and Technology, Port Harcourt, Nigeria.

KashifSR, Yasee M, Arshad M, Ayub M (2008). Response of okra (Hibiscus esculentus L.) to soil given encapsulated calcium carbide. Pakistan Journal ofBotany 40(1):175-181.

Odeleye FO, Odeleye OMO, Dada OA (2007). The performance of soybean (Glycine max L.) under varying weeding regimes in South Western Nigeria. Department of Crop Protection and Environmental Biology, University Of Ibadan, Ibadan, Nigeria; National Horticultural Research Institute, Jericho,Idi-Ishin, Ibadan, Olabisionabanjo.

Olorunmaiye KS, Ogunfolaji RT (2002). Effect of density and duration of Euphorbia heterophylla L. on the performance of cowpea Vigna unguiculata Walp. Journal of Nigeria Society for Experimental Biology 2(1):17-22.

PaththinigeSS, Upashantha PSG, Banda RMR, Fonseka RM (2008).Effect of plant spacing on yield and fruit characteristics of okra (Abelmoschus esculentus). Tropical Agricultural Research 28:336-342.

Poggio SL (2005). Structure of weed communities occurring in monoculture and intercropping of field pea and barley. Agricultural Ecosystem andEnvironment 109(1-2):48-58. 
Rao VS (2000). Weed management in crop and plantation crops. Principles of weed science (2 ${ }^{\text {nd }}$ edition) pp 211-216.

Reddy KN, Whiting KM (2002). Weed control and economic comparisons in soybean planting systems. Journal of Sustainable Agriculture 21(2):21-35.

Reddy KN, Whiting KM (2000). Weed control and economic comparisons of Glyphosate resisitant, sulfonylena-tolerant, and conventional soybean (Glycine max) systems. Weed Technology 14(1):204-211.

Rizzardi MA (2004). Interference of populations of Euphorbia heterophylla and Ipomoea ramosissima isolated or in mixture in soybean crop. Planta Daninha 22(1):29-34.
Saifullah M, Rabbani MG (2009). Evaluation and characterization of okra (Abelmoschus esculentus L. Moench.) genotypes. Journal of Agriculture 7(1):92-99.

Saka HO, Hassan OU, Adekola FO, Olorunmaiye KS (2013). Effect of weed density (Euphorbia heterophylla) on the performance of popcorn maize (Zeamays var. everta). Agronomskiglasnik 75(5-6):267-278.

Smith MAK, IK Ojo (2007). Influence of intra-row spacing and weed management system on colonization of weed, pot yield and quality in okra African CropScience Conference Proceeding 8(1):313-317. 